\title{
Quantitative Susceptibility Mapping (QSM) Algorithms: Mathematical Rationale and Computational Implementations
}

\author{
Youngwook Kee, Zhe Liu, Liangdong Zhou, Alexey Dimov, Junghun Cho, Ludovic de Rochefort, \\ Jin Keun Seo, and Yi Wang*, Fellow, IEEE
}

\begin{abstract}
Quantitative susceptibility mapping (QSM) solves the magnetic field-to-magnetization (tissue susceptibility) inverse problem under conditions of noisy and incomplete field data acquired using magnetic resonance imaging. Therefore, sophisticated algorithms are necessary to treat the ill-posed nature of the problem and are reviewed here. The forward problem is typically presented as an integral form, where the field is the convolution of the dipole kernel and tissue susceptibility distribution. This integral form can be equivalently written as a partial differential equation (PDE). Algorithmic challenges are to reduce streaking and shadow artifacts characterized by the fundamental solution of the PDE. Bayesian maximum a posteriori (MAP) estimation can be employed to solve the inverse problem, where morphological and relevant biomedical knowledge (specific to the imaging situation) are used as priors. As the cost functions in Bayesian QSM framework are typically convex, solutions can be robustly computed using a gradient-based optimization algorithm. Moreover, one can not only accelerate Bayesian QSM, but also increase its effectiveness at reducing shadows using prior knowledge based precondition. Improving the efficiency of QSM is under active development, and a rigorous analysis of preconditioning needs to be carried out for further investigation.
\end{abstract}

Index Terms-Bayesian image reconstruction, deconvolution, nonsmooth convex optimization, partial differential equations, preconditioning, quantitative susceptibility mapping (QSM).

\section{INTRODUCTION}

Q UANTITATIVE susceptibility mapping (QSM) [1, 2] is a noninvasive magnetic resonance imaging (MRI) method that enables quantitative investigation of the tissue magnetic susceptibility. QSM is based on the post-processing of the magnetic field map derived from the phase data of gradient echo (GRE) MRI that is readily available on most MRI scanners [3]. QSM has been applied to a wide range of biomedical problems [1, 4-9] including: 1) demyelination, inflammation, and iron overload in multiple sclerosis [1017], 2) neurodegeneration and iron overload in Alzheimer's disease [18-20], Parkinson's disease [21-33] and Huntington's disease [34], 3) changes in metabolic oxygen consumption

Y. Kee and L. Zhou are with the Department of Radiology, Weill Cornell Medical College, New York, NY, 10065 USA.

Z. Liu, A. Dimov, and J. Cho are with the Department of Biomedical Engineering, Cornell University, Ithaca, NY, 14853 USA.

L. de Rochefort is with Aix-Marseille University, CNRS, CRMBM UMR 7339, Marseille, France.

J. K. Seo is with the Department of Computational Science and Engineering, Yonsei University, Seoul, South Korea.

Y. Wang is with the Department of Radiology, Weill Cornell Medical College, New York, NY, 10065 USA and is with the Department of Biomedical Engineering, Cornell University, Ithaca, NY, 14853 USA.

*Corresponding Author (email: yiwang@med.cornell.edu).

Manuscript received March 16, 2017.
[35-41], 4) hemorrhage including microhemorrhage and blood degradation [42-47], 5) bone mineralization [48], and 6) drug delivery using magnetic nanocarriers [49, 50]. QSM of dominant scalar susceptibility sources in tissue (including iron and calcium) has now become reasonably robust and accurate for routine use in research and practice [1, 7].

Algorithmically, QSM solves a challenging ill-posed inverse problem. QSM was coined from a breakthrough in 2008 using the Bayesian maximum a posteriori (MAP) estimation theory [2, 51-54]. Yet, it remains to be fully developed and there is plenty of room for further optimization. To establish this milestone and characterize associated challenges, this review paper defines the forward problem in QSM as a partial differential equation (PDE). Then, the difficulties of the field-to-susceptibility inversion problem are systematically analyzed based on general solutions for the PDE. This analysis emphasizes the limitations of early attempts [55-58] compared to Bayesian approaches. Bayesian QSM methods optimally estimate the underlying tissue susceptibility distribution from noisy and incomplete magnetic field data (likelihood) using tissue structural information (a priori). To this end, variational methods are used to minimize a cost function associated with the negative posterior, where the optimal solution (MAP estimate) corresponds to a minimizer of the cost function. In Bayesian QSM, such a cost function is typically given as a convex objective so that one can use solvers developed in modern convex optimization [59] for efficient computation of a solution. This principled approach avoids the drawbacks inherent in the non-Bayesian attempts and has laid the foundation for QSM, generating a great deal of interest in studying signal phase, the often-neglected other half of the complex MRI signal [60-75]

There are a number of existing review papers on QSM, which can be categorized as follows: 1) General reviews [4, 5, 9, 76], 2) phase processing methods [77, 78], 3) biophysics [1], and 4) clinical applications [7, 8]. Unlike these, this review focuses on mathematical and computational aspects of the deconvolution problem in QSM. Specifically, the source of artifacts in QSM images is identified as dipoleincompatible fields that deviates from the field generated by dipoles. Then, the artifacts are characterized as streaking and shadow by the fundamental solution of a wave-type operator. This characterization establishes a rationale for the design principles of a regularization term in the Bayesian QSM methods, implying that early attempts based on k-space truncation causes additional dipole-incompatibility in the field. These Bayesian QSM methods require numerical optimization whose implementation details and preconditioning techniques 
are provided here. Lastly, the review discusses remaining challenges in this field. This review will serve as a unique reference to QSM algorithm developers working on numerical optimization.

\section{ForWARD PROBLEM-BIOPHYSICS MODEL}

In an MRI scanner with a main static magnetic field $\mathbf{B}_{\mathbf{0}}=\left(0,0, B_{0}\right)$, tissues gain a magnetization distribution $\mathbf{M}(\mathbf{r})$, where $\mathbf{r}=(x, y, z) \in \mathbb{R}^{3}$ is a position vector. This magnetization generates a magnetic field perturbation $\mathfrak{B}(\mathbf{r})$ superpositioning on $\mathbf{B}_{\mathbf{0}}$ according to magnetostatic macroscopic Maxwell's equations [79]

$$
\begin{aligned}
\nabla \cdot \mathfrak{B}(\mathbf{r}) & =0 \\
\nabla \times \mathfrak{B}(\mathbf{r}) & =\mu_{0} \nabla \times \mathbf{M}(\mathbf{r}),
\end{aligned}
$$

where $\mu_{0}$ is the vacuum permittivity. Using the Lorentz sphere correction model [80, 81], the macroscopic field $\mathfrak{B}(\mathbf{r})$ can be related to the microscopic field $\mathbf{b}_{\ell}(\mathbf{r})$ experienced by the spins of water protons in tissue, the MRI signal generators, as follows

$$
\mathbf{b}_{\ell}(\mathbf{r})=\mathfrak{B}(\mathbf{r})-\frac{2}{3} \mu_{0} \mathbf{M}(\mathbf{r}) .
$$

MRI signal phase can be used for this field estimation, which includes nonlinear data fitting and field unwrapping as summarized in recent reviews [1, 76]. Applying the curl operation to both sides of (2) and using (1) and (3), we obtain

$$
-\Delta\left(\mathbf{b}_{\ell}-\mathbf{B}_{\mathbf{0}}\right)=\mu_{0}\left(\nabla(\nabla \cdot \mathbf{M})-\frac{1}{3} \Delta \mathbf{M}\right),
$$

where $\Delta$ is the Laplacian operator. The Fourier transform of (4), denoted as $\mathcal{F}$, leads us to

$$
\begin{array}{rl}
|\mathbf{k}|^{2} & \mathcal{F}\left[\mathbf{b}_{\ell}-\mathbf{B}_{\mathbf{0}}\right](\mathbf{k}) \\
\quad=\mu_{0}\left(\mathbf{k}(\mathbf{k} \cdot \mathcal{F}[\mathbf{M}](\mathbf{k}))-\frac{1}{3}|\mathbf{k}|^{2} \mathcal{F}[\mathbf{M}](\mathbf{k})\right),
\end{array}
$$

where $\mathbf{k}=\left(k_{x}, k_{y}, k_{z}\right) \in \mathbb{R}^{3}$ is a spatial frequency vector. Isotropic biological tissue concerned here has weak magnetic susceptibility, i.e., $|\chi| \ll 1$, hence its magnetization can be expressed as $\mathbf{M}=\left(0,0, M_{z}\right)$, where $M_{z} \simeq\left(B_{0} / \mu_{0}\right) \chi$. In the following, we define the relative difference field (RDF) $b$, which is referred to as field, total field 1 , or field inhomogeneity:

$$
b(\mathbf{r}):=\frac{b_{\ell z}(\mathbf{r})-B_{0}}{B_{0}},
$$

where $b_{\ell z}$ denotes the $z$-component of $\mathbf{b}_{\ell}$. We can rewrite (5) in terms of the $z$-component as follows

$$
|\mathbf{k}|^{2} B(\mathbf{k})=\left(\frac{k_{x}^{2}+k_{y}^{2}-2 k_{z}^{2}}{3}\right) X(\mathbf{k}),
$$

where $B(\mathbf{k}):=\mathcal{F}[b](\mathbf{k})$ and $X(\mathbf{k}):=\mathcal{F}[\chi](\mathbf{k})$. For all $\mathbf{k} \neq \mathbf{0}$, we can rewrite the expression (6) as

$$
B(\mathbf{k})=\left(\frac{1}{3}-\frac{k_{z}^{2}}{|\mathbf{k}|^{2}}\right) X(\mathbf{k}):=D(\mathbf{k}) X(\mathbf{k}),
$$

\footnotetext{
${ }^{1}$ Note that the acronym RDF has been often referred to as tissue (local) field $(:=$ total field - background field) in the literature. In this paper, however, RDF means total field by its definition.
}

where $D(\mathbf{k})=1 / 3-k_{z}^{2} /|\mathbf{k}|^{2}$ is the dipole kernel in $\mathbf{k}$-space. Equivalently,

$$
b(\mathbf{r})=\lim _{\epsilon \searrow 0} \int_{\left|\mathbf{r}-\mathbf{r}^{\prime}\right|>\epsilon} d\left(\mathbf{r}-\mathbf{r}^{\prime}\right) \chi\left(\mathbf{r}^{\prime}\right) \mathrm{d} \mathbf{r}^{\prime},
$$

where $d$ denotes the dipole kernel in $\mathbf{r}$-space (image space) defined as

$$
d(\mathbf{r}):=\mathcal{F}^{-1}[D](\mathbf{r})=\frac{2 z^{2}-x^{2}-y^{2}}{4 \pi|\mathbf{r}|^{5}} .
$$

This biophysics model (forward problem) describes that an RDF is generated by the dipole kernel convolved with the magnetic susceptibility distribution $\chi$.

\section{InVERse Problem-A WaVe Propagation VieW}

Current acquisition and phase processing methods allow us to estimate an RDF from GRE phase data with high precision. The problem is to recover $\chi$ from the RDF $b$ by fitting this with the forward model (7) in k-space or (8) in image space. This leads to solving a deconvolution problem (inverse problem) whose nature is ill-posed because of a conic surface defined by

$$
\Gamma_{0}:=\left\{\mathbf{k} \in \mathbb{R}^{3} \mid k_{x}^{2}+k_{y}^{2}-2 k_{z}^{2}=0\right\} .
$$

The surface $\Gamma_{0}$ is spanned by the magic angle $\left(\simeq 54.7^{\circ}\right)$ with respect to the $k_{z}$-direction as shown in Fig. 11 As can be seen in (6), the $\mathrm{k}$-space points in $\Gamma_{0}$ cause division by zero when the deconvolution is performed in $\mathrm{k}$-space.

This ill-posed inverse problem can be investigated in image space. Let us consider (4), the partial differential equation (PDE) associated with (6), as follows:

$$
P(\partial) \chi(\mathbf{r})=-\Delta b(\mathbf{r}),
$$

where $P(\partial)$ is a wave-type operator (propagator) given as

$$
P(\partial):=-\frac{1}{3} \Delta+\frac{\partial^{2}}{\partial z^{2}}
$$

Because (9) is the inverse Fourier transform of (6), the inverse problem of recovering $X(\mathbf{k})$ in 6 may be viewed as the $\mathbf{k}$ space representation of the PDE (9). This PDE is a wave-type equation in $\chi$, where the $z$-axis is considered time, but is a Laplace equation in $b$. As noted in [82], one good strategy for investigating any PDE is to identify its explicit solutions; this can be achieved by deriving a fundamental solution. Since every constant coefficient linear PDE on $\mathbb{R}^{n}$ has a fundamental solution [83], a solution of (9]) denoted as $\chi^{\sharp}$ can be expressed as

$$
\chi^{\sharp}=g *(-\Delta b)=-\int_{\mathbb{R}^{3}} g\left(\mathbf{r}-\mathbf{r}^{\prime}\right) \Delta_{\mathbf{r}^{\prime}} b\left(\mathbf{r}^{\prime}\right) \mathrm{d} \mathbf{r}^{\prime},
$$

where $\mathbf{r}^{\prime}=\left(x^{\prime}, y^{\prime}, z^{\prime}\right) \in \mathbb{R}^{3}$ is a position vector and

$$
g(\mathbf{r})=\left\{\begin{array}{cl}
\frac{3}{4 \pi \sqrt{z^{2}-2\left(x^{2}+y^{2}\right)}} & \text { if } \mathbf{r} \in \Upsilon ; \\
0 & \text { otherwise }
\end{array}\right.
$$

is the fundamental solution of the differential operator $P(\partial)$ with $\mathcal{F}[g](\mathbf{k})=\frac{3}{4 \pi^{2}\left(k_{x}^{2}+k_{y}^{2}-2 k_{z}^{2}\right)}=: \frac{1}{P(\mathbf{k})}$ as displayed in Fig. 1 and $\Upsilon$ defined as

$$
\Upsilon:=\left\{\mathbf{r} \in \mathbb{R}^{3} \mid z^{2}-2\left(x^{2}+y^{2}\right)>0\right\} .
$$




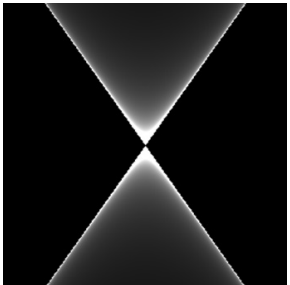

$g(\mathbf{r})$

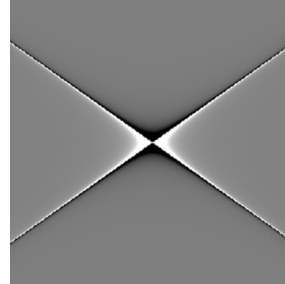

$1 / P(\mathbf{k})$

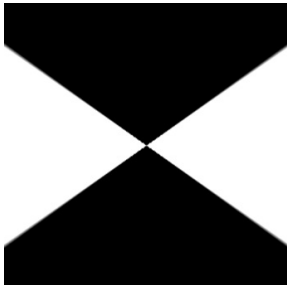

$D(\mathbf{k})$
Fig. 1. The fundamental solution $g(\mathbf{r})$ is displayed on the left with the window level $[0,5]$ in sagittal view. Notice that the conic surface is spanned by $\sim 35.3^{\circ}$. Its Fourier transform $\mathcal{F}[g](\mathbf{k})=1 / P(\mathbf{k})=1 /\left(4 \pi|\mathbf{k}|^{2} D(\mathbf{k})\right)$ is displayed in the middle with the window level $[-100,100]$ in sagittal view. The dipole kernel $D(\mathbf{k})$ is displayed on the right with the window level $[-0.01,0.01]$ in sagittal view. Here, the conic surface is spanned by $\sim 54.7^{\circ}$. Observe that the conic surface of the fundamental solution is perpendicular to that of $D(\mathbf{k})$.

The detailed derivation of (11) can be found in Appendix 0.3 in [84]. Note that $\Upsilon$ defines a double-cone in $\mathbb{R}^{3}$ whose conic surface is spanned by the angle $\simeq 35.3^{\circ}$, the complimentary magic angle. The fundamental solution $g$ is shown in Fig. 1.

We can also obtain from (7), the Fourier transform of (9), a solution in $\mathbf{k}$-space as follows:

$$
X(\mathbf{k})=B(\mathbf{k}) / D(\mathbf{k}), \quad \mathbf{k} \in \mathbb{R}^{3} \backslash\{\mathbf{0}\} .
$$

How do we interpret these two explicit forms- 10 and (12) — of a solution to the field-to-source inversion problem, particularly when there is some perturbation, e.g., noise, in the RDF $b$ ?

\section{A. Dipole-Compatible Field Data}

Suppose that RDF data is "purely" generated by dipoles (denoted here as $b_{c}(\mathbf{r}), B_{c}(\mathbf{k})$,), i.e.,

$$
B_{c}(\mathbf{k})=\frac{P(\mathbf{k}) X_{c}(\mathbf{k})}{4 \pi^{2}|\mathbf{k}|^{2}}=D(\mathbf{k}) X_{c}(\mathbf{k}), \quad \mathbf{k} \in \mathbb{R}^{3} \backslash\{\mathbf{0}\}
$$

and we assign some constant to $B_{c}(\mathbf{0})$. Then, the field $B_{c}$ is said to be dipole compatible. For any $B_{c}$, one can perfectly recover $X_{c}$ for $\mathbf{k} \in \mathbb{R}^{3} \backslash \Gamma_{0}$ by the relation

$$
X_{c}(\mathbf{k})=\frac{B_{c}(\mathbf{k})}{D(\mathbf{k})}=\frac{4 \pi^{2}|\mathbf{k}|^{2} B_{c}(\mathbf{k})}{P(\mathbf{k})} .
$$

For $\mathbf{k} \in \Gamma_{0} \backslash\{\mathbf{0}\}, X_{c}$ is recovered by the following limit [85]:

$$
X_{c}(\mathbf{k})=\lim _{h \searrow 0} \frac{4 \pi^{2}\left|\mathbf{k}+h \mathbf{e}_{z}\right|^{2} B_{c}\left(\mathbf{k}+h \mathbf{e}_{z}\right)}{P\left(\mathbf{k}+h \mathbf{e}_{z}\right)}
$$

where $\mathbf{e}_{z}=(0,0,1)$. It should be noted that neither $\lim _{\mathbf{k} \rightarrow \mathbf{0}} X_{c}(\mathbf{k})$ nor $X_{c}(\mathbf{0})$ exist. This can easily be shown by considering two different paths (such as $k_{x}=k_{y}=0, k_{z} \rightarrow 0$, then $1 / D \rightarrow-3 / 2$ or $k_{x}=k_{z}=0, k_{y} \rightarrow 0$, then $1 / D \rightarrow 3$ ). Therefore, $X_{c}$ is only recovered up to a constant shift, i.e., $X_{c}(\mathbf{0})$, by using the direct reconstruction formulas (14) and (15). Note that $X_{c}(\mathbf{0})$ is a symbolic notation for some constant shift in image space. The performance of (14) and (15) is shown in Fig. 2 where we assume that $X_{c}(\mathbf{0})=\mathbf{0}$.

Remark 1: That $X_{c}(\mathbf{k})$ is undefined at the center of $\mathbf{k}$ space can be also checked from the PDE (9): We see that

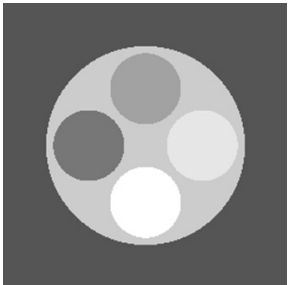

Reference $\chi_{c}$

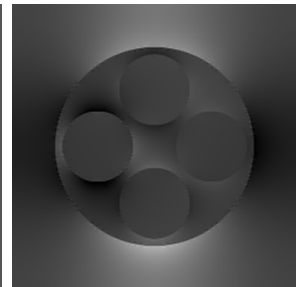

$b_{c}$

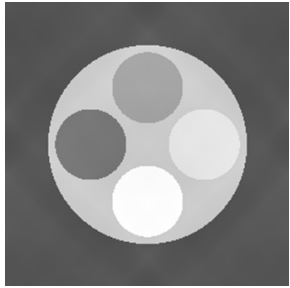

Recovered $\chi_{c}$
Fig. 2. The dipole compatible field $b_{c}$ displayed in the middle with the window level $[-0.3,1]$ was generated from the dipole model [13] using the reference $\chi_{c}$ shown on the left with $[-0.5 \mathrm{ppm}, 1 \mathrm{ppm}]$. We recovered $\chi_{c}$ shown on the right with $[-0.5 \mathrm{ppm}, 1 \mathrm{ppm}]$ using [14] and [15]. Here, the largest sphere is $0.7 \mathrm{ppm}$ and the background (outside the sphere) is $0 \mathrm{ppm}$. The four spheres inside the largest one, have susceptibility values of $1,0.85$, 0.45 , and $0.2 \mathrm{ppm}$, going counterclockwise starting from the bottom.

$P(\partial)(\chi(\mathbf{r})+C)=P(\partial) \chi(\mathbf{r})=-\Delta b(\mathbf{r})$ for any constant $C$. Hence, susceptibility values are often reported with respect to an estimate of reference tissue susceptibility. This is also reflected in (8) that $\lim _{\epsilon \searrow 0} \int_{\left|\mathbf{r}-\mathbf{r}^{\prime}\right|>\epsilon} C d\left(\mathbf{r}^{\prime}\right) \mathrm{d} \mathbf{r}^{\prime}=0$.

\section{B. Dipole-Incompatible Field Data-Streaking and Shadow}

Let $\Gamma_{0}^{B}$ be the set $\left\{\mathbf{k} \in \Gamma_{0} \mid B(\mathbf{k}) \neq 0\right\} \subseteq \Gamma_{0} \backslash\{\mathbf{0}\}$. Then, regarding the dipole-compatible part, we see $\Gamma_{0}^{B_{c}}=\emptyset$ since $B_{c}(\mathbf{k})=D(\mathbf{k}) X_{c}(\mathbf{k})$ where $D(\mathbf{k})=0$ in $\Gamma_{0} \backslash\{\mathbf{0}\}$. Let $B_{i}$ be a measured field such that $\Gamma_{0}^{B_{i}} \neq \emptyset$; then we call such a field dipole-incompatible as the field cannot be expressed by the dipole model (13). Consider a dipole-compatible field with additive noise, $B_{i}(\mathbf{k})=D(\mathbf{k}) X(\mathbf{k})+N(\mathbf{k})$, where $N(\mathbf{k})$ is (Gaussian) noise in $\mathbf{k}$-space. Then, we express the underlying susceptibility $\chi_{i}$ in terms of the fundamental solution $g$ in (10) and $(11)$ as follows:

$$
\chi_{i}(\mathbf{r})=\left(g *\left(-\Delta b_{i}\right)\right)(\mathbf{r})=\int_{\mathbb{R}^{3}} \frac{-3 \Delta_{\mathbf{r}^{\prime}} b_{i}\left(\mathbf{r}^{\prime}\right)}{4 \pi \sqrt{E\left(\mathbf{r}, \mathbf{r}^{\prime}\right)}} \mathrm{d} \mathbf{r}^{\prime},
$$

where $E\left(\mathbf{r}, \mathbf{r}^{\prime}\right):=\left(z-z^{\prime}\right)^{2}-2\left(\left(x-x^{\prime}\right)^{2}+\left(y-y^{\prime}\right)^{2}\right)>0$ defines the double-cone $\Upsilon$ above and below the incompatible source $-\Delta_{\mathbf{r}^{\prime}} b_{i}\left(\mathbf{r}^{\prime}\right)$. Strong propagation of $-\Delta_{\mathbf{r}^{\prime}} b_{i}\left(\mathbf{r}^{\prime}\right)$ is present along the double-cone surface where $E\left(\mathbf{r}, \mathbf{r}^{\prime}\right)$ vanishes, which manifests as streaking in sagittal and coronal views and ringing in the axial view.

To see this more concretely, suppose that an incompatible source is expressed as a linear combination of the Dirac delta functions, i.e., $-\Delta b_{i}(\mathbf{r})=\sum_{j=1}^{N} c_{j} \delta\left(\mathbf{r}-\mathbf{r}_{j}^{\prime \prime}\right)$, where $c_{j} \in \mathbb{R}$ and $\mathbf{r}_{j}^{\prime \prime}=\left(x_{j}^{\prime \prime}, y_{j}^{\prime \prime}, z_{j}^{\prime \prime}\right) \in \mathbb{R}^{3}$ is a position vector. Therefore, $\chi_{i}$ is expressed as a linear combination of Green's functions, where each of the functions defines the double-cone $\Upsilon$ located at $\mathbf{r}_{1}^{\prime \prime}, \ldots, \mathbf{r}_{N}^{\prime \prime}$ as follows:

$$
\chi_{i}(\mathbf{r})=g(\mathbf{r}) * \sum_{j=1}^{N} c_{j} \delta\left(\mathbf{r}-\mathbf{r}_{j}^{\prime \prime}\right)=\sum_{j=1}^{N} c_{j} g\left(\mathbf{r}-\mathbf{r}_{j}^{\prime \prime}\right) .
$$

Notice that the singular support of the double-cone $\Upsilon$ defined as the set of $\mathbf{r} \in \mathbb{R}^{3}$ such that $z^{2}-2\left(x^{2}+y^{2}\right)=0$ forms a conic surface spanned by the complimentary magic angle $\left(\simeq 35.3^{\circ}\right)$ along the $z$-axis. This conic surface is associated with $\Gamma_{0}$ defined in $\mathbf{k}$-space, a zero cone surface spanned by the 


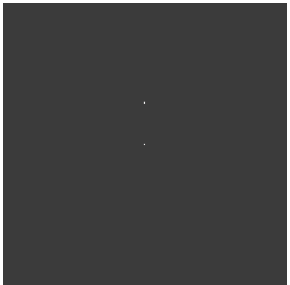

$b_{i}$ in axial

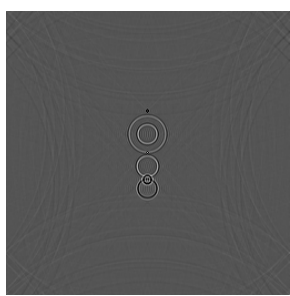

$\chi_{i}$ in axial

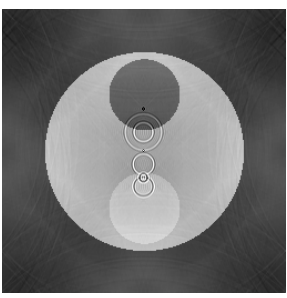

$\chi_{c}+\chi_{i}$ in axial

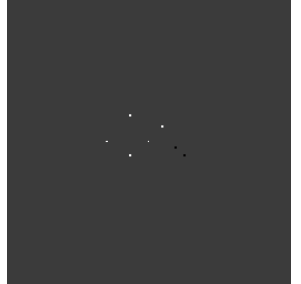

$b_{i}$ in sagittal

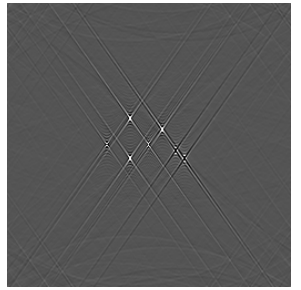

$\chi_{i}$ in sagittal

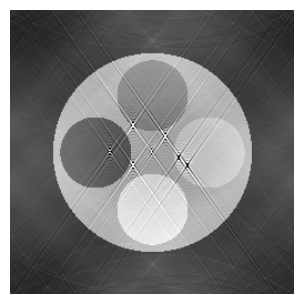

$\chi_{c}+\chi_{i}$ in sagittal

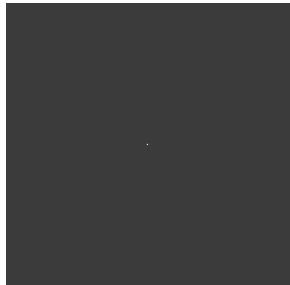

$b_{i}$ in coronal

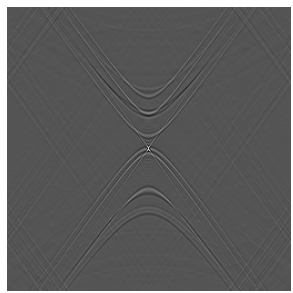

$\chi_{i}$ in coronal

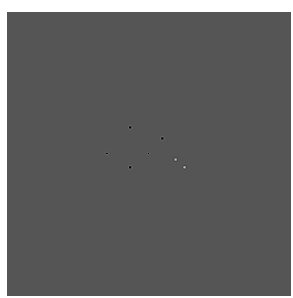

$\Delta b_{i}$ in sagittal

Fig. 3. The top row shows a dipole-incompatible field $b_{i}$ (point sources) with the window level $[-0.3,1]$; the susceptibility values of white and black dots are +1 and -1 , respectively. The rightmost image in the bottom row is the Laplacian of $b_{i}$ in sagittal with the window level $[-0.5,1]$. The middle row shows the recovered susceptibility $\chi_{i}$ with the window level $[-0.5 \mathrm{ppm}, 1 \mathrm{ppm}]$. Artifacts manifest as ringing in the axial view and streaking in sagittal and coronal views. The leftmost and middle panels in the bottom row show the recovered map with the window level $[-0.5 \mathrm{ppm}, 1 \mathrm{ppm}]$ from the RDF $b_{c}+b_{i}$ where $b_{c}$ is given as in Fig. 2 Susceptibility was reconstructed using TKD [58] with 0.005 as the value of truncation.

magic angle $\left(\simeq 54.7^{\circ}\right)$ with respect to the $k_{z}$-axis as shown in Fig. 1. Note that the two cone surfaces meet at a right angle. Hence, strong streaking in sagittal and coronal views with the angle $35.3^{\circ}$ from the $z$-axis is observed as demonstrated in Fig. 3 .

Remark 2: Observe that $E\left(\mathbf{r}, \mathbf{r}^{\prime}\right)$ in the denominator of (16) that causes propagating artifacts does not come into play when the field is dipole-compatible. As can be seen in the direct reconstruction formulas (14) and $[15,1 / P(\mathbf{k})=\mathcal{F}[g](\mathbf{k})$ is effectively canceled out when the field is dipole-compatible. This observation reassures the existence of streaking-free solutions proved in [84] 85].

Not only is streaking explained by (16), but also the expression allows $\chi_{i}$ to have other types of "apparent artifacts". Suppose that $\Delta b_{i}$ is smoothly distributed across the image volume; then, $\chi_{i}$ is expected to exhibit slowly varying spatial artifacts that manifest as dark shadows as demonstrated in Fig. 4 Note that if the sign of $\Delta b_{i}$ is flipped, bright clouds will be seen instead. Although these artifacts appear different with low-frequency components as opposed to streaking with highfrequency, it is crucial to keep in mind that the expression (16) remains the same in both cases. In other words, dark shadows

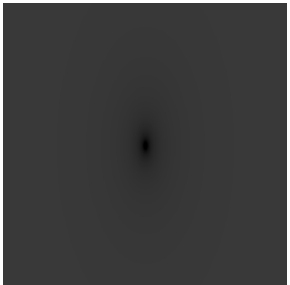

$b_{i}$ in axial

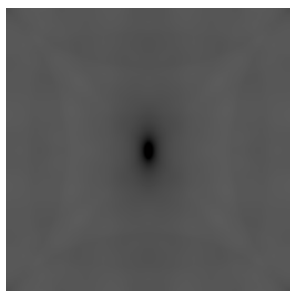

$\chi_{i}$ in axial

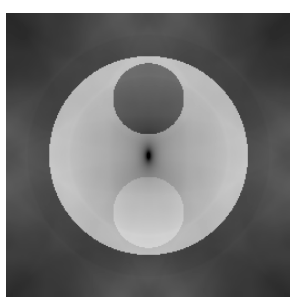

$\chi_{c}+\chi_{i}$ in axial

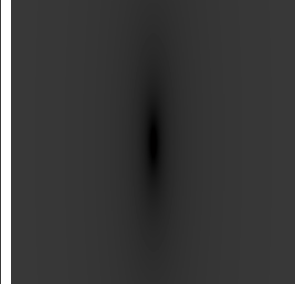

$b_{i}$ in sagittal

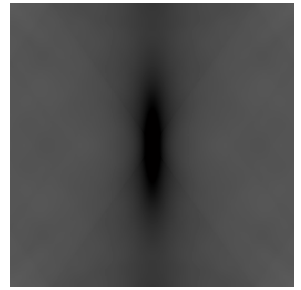

$\chi_{i}$ in sagittal

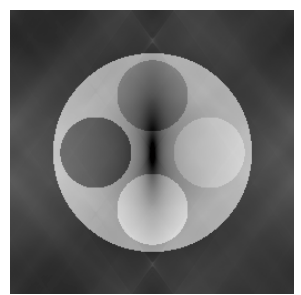

$\chi_{c}+\chi_{i}$ in sagittal

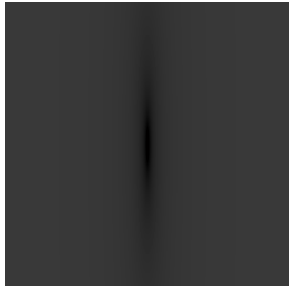

$b_{i}$ in coronal

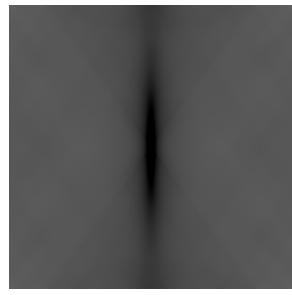

$\chi_{i}$ in coronal

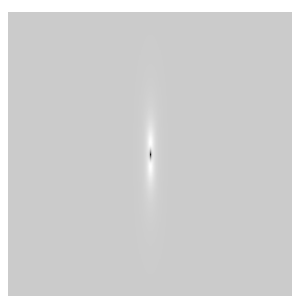

$\Delta b_{i}$ in sagittal
Fig. 4. The top row shows a dipole-incompatible field $b_{i}$ (smooth distribution) with the window level $[-0.3,1]$; the values of susceptibility distribution that generates the field $b_{i}$ are all negative. The rightmost image in the bottom row is the Laplacian of $b_{i}$ with the window level $[-0.5,1]$. The middle row shows the recovered susceptibility $\chi_{i}$ with the window level $[-0.5 \mathrm{ppm}, 1 \mathrm{ppm}]$. Artifacts manifest as dark shadows. The leftmost and middle panels in the bottom row show the recovered map with the window level $[-0.5 \mathrm{ppm}, 1 \mathrm{ppm}]$ from the RDF $b_{c}+b_{i}$ where $b_{c}$ is given as in Fig. 2 Susceptibility was reconstructed using TKD [58] with 0.005 as the value of truncation.

are simply a superposition of the double-cones $\Upsilon$ computed by $(16)$.

How does a solution expressed as (10) behave? If a given $\mathrm{RDF}$ is dipole-compatible, perfect recovery is possible up to a constant shift using (14) and (15). If the RDF is dipoleincompatible, streaking and shadow artifacts will be present according to 16 and the shape of dipole-incompatible source distributions.

\section{Minimizing Streaking and Shadow: Early Attempts based on $\mathbf{k}$-space truncation versus Bayesian MAP Estimation}

Dipole-incompatible field data that deviates from the dipole model (13) includes noise, anisotropic sources, chemical shift, or discretization error. Projecting or filtering out the incompatible part directly from the GRE data is very difficult, because 1) noise is omnipresent in practice, 2) anisotropic sources cannot be measured reliably in a single-angle acquisition, and 3) discretization is indispensable for numerical computation. Consequently, the formulas (14) and (15) cannot be used in practice, as their use on the MRI estimated field data causes severe artifacts from the dipole-incompatible part. 
Early attempts to solve the inverse problem are based on kspace truncation to modify (13) near the zero-cone $\Gamma_{0}$ where $1 / D(\mathbf{k}) \rightarrow \infty$ for $\mathbf{k}$ near $\Gamma_{0}$. These methods include truncated k-space division (TKD) [58], truncated singular value decomposition (TSVD) or Tikhonov-regularized minimal norm (MN) [53, 63], iterative susceptibility weighted imaging and susceptibility mapping (iSWIM) [86], and analytic continuation (AC) [87]. Truncation in $\mathbf{k}$-space changes the dipole kernel near the zero-cone $\Gamma_{0}$, which is equivalent to adding dipole-incompatible field components. Consequently, k-space truncation causes additional streaking according to (16), and is demonstrated in Fig. 5 .

Remark 3: Observe that in Fig. 5. strong streaking appears at the interface between two different homogeneous regions (jump set). A rigorous analysis on this type of streaking can be found in [85. 88] where the notion of wave front set [89] plays a crucial role in identifying its appearance.

Bayesian inference approaches overcome the limitations of $\mathbf{k}$-space truncation by directly penalizing streaking and shadow in image space, which can be solved by a variational method. In this framework, one seeks an optimal solution that minimizes

$$
E(\chi):=E_{\text {data }}(b \mid \chi)+\lambda E_{\text {reg }}(\chi),
$$

where the data fidelity term $E_{\text {data }}$ measures the disagreement between $\chi$ and $b$, and the regularization term $E_{\text {reg }}$ that encodes additional information measures a certain property of $\chi$, e.g., smoothness, sparsity, and consistency with known morphologic and biologic information. The regularization parameter $\lambda>0$ balances the two terms, which can be empirically chosen by the L-curve heuristic [90, 91]. Note that this variational method is intrinsically connected to Bayesian MAP estimation [92], i.e., $p(b \mid \chi)=e^{-E_{\text {data }}(b \mid \chi)}$ and $p(\chi)=e^{-E_{\text {reg }}(\chi)}$. If the objective $E(\chi)$ is convex, its globally optimal solution (MAP estimate) can be computed by solving the corresponding Euler-Lagrange equation-whose solutions are functions for which $E(\chi)$ is stationary. Note that writing out an explicit solution for the Euler-Lagrange equation is typically impossible, making numerical optimization required to compute a numerical solution. In general, the data fidelity term is derived from the forward problem; in most cases it is of the form $\|d * \chi-b\|_{w}^{2}$, a weighted 2-norm. However, there are a wide range of variations in the regularization term $E_{\text {reg }}$ to reduce streaking and shadows, generating various Bayesian QSM algorithms as outlined in the next section.

\section{BAYESIAN QSM METHODS}

In this section, we give an overview of Bayesian QSM methods based on an archetypal model. These methods are capable of reducing both streaking and shadow when proper priors are employed-which is under active development.

\section{A. Reducing Streaking-Morphology Enabled Dipole Inver- sion (MEDI)}

The morphology-enabled dipole inversion (MEDI) method [72] is an archetypal model that has been widely used for

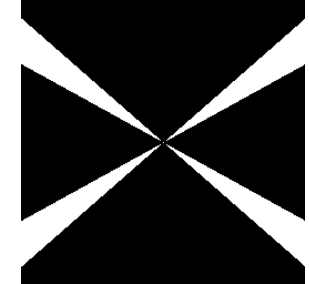

$\eta_{\Gamma_{\epsilon_{1}}}$

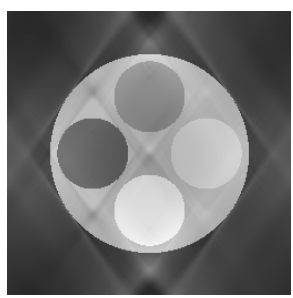

$\chi_{\epsilon_{1}}$

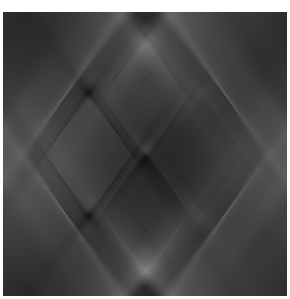

$\chi_{\epsilon_{1}}-\chi_{c}$

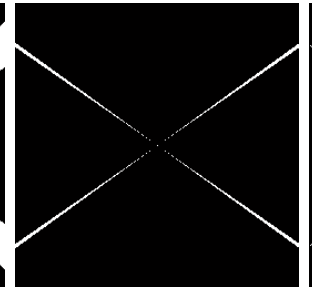

$\eta_{\Gamma_{\epsilon_{2}}}$

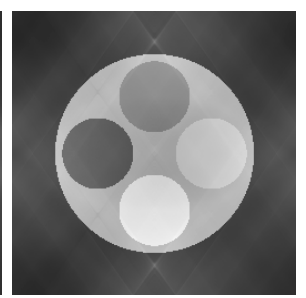

$\chi_{\epsilon_{2}}$

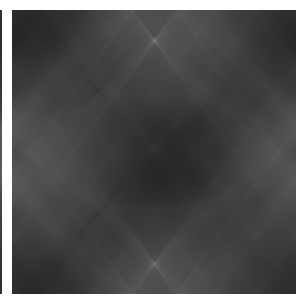

$\chi_{\epsilon_{2}}-\chi_{c}$

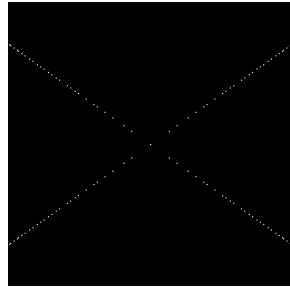

$\eta_{\Gamma_{\epsilon_{3}}}$

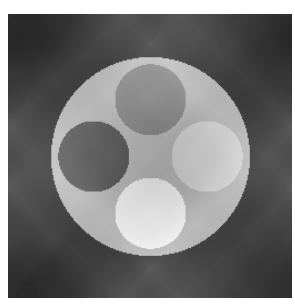

$\chi_{\epsilon_{3}}$

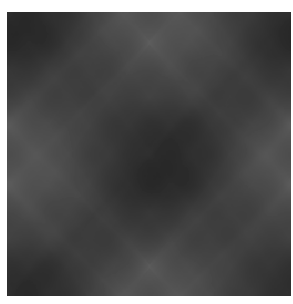

$\chi_{\epsilon_{3}}-\chi_{c}$
Fig. 5. The top row shows the indicator function of $\Gamma_{\epsilon}$ denoted by $\eta_{\Gamma_{\epsilon}}$, where $\Gamma_{\epsilon}$ is defined as $\left\{\mathbf{k} \in \mathbb{R}^{3}:|D(\mathbf{k})|<\epsilon\right\}$ with different values of $\epsilon$, where $\epsilon_{1}=0.1, \epsilon_{2}=0.01$, and $\epsilon_{3}=0.001$. That is, $\eta_{\Gamma_{\epsilon}}(\mathbf{k})=1$ for $\mathbf{k} \in \Gamma_{\epsilon}$ and $\eta_{\Gamma_{\epsilon}}(\mathbf{k})=0$ for $\mathbf{k} \in \mathbb{R}^{3} \backslash \Gamma_{\epsilon}$, i.e., white $=1$ and black $=0$. For the dipole-compatible field described in Fig. 2. the second row shows the corresponding TKD solutions defined as $\chi_{\epsilon}=\mathcal{F}^{-1}\left(B_{c}(\mathbf{k}) / D(\mathbf{k})(1-\right.$ $\left.\left.\left.\eta_{\Gamma_{\epsilon}}(\mathbf{k})\right)\right)=\chi_{c}-\mathcal{F}^{-1}\left(B_{c}(\mathbf{k}) / D(\mathbf{k})\right) * \mathcal{F}^{-1}\left(\eta_{\Gamma_{\epsilon}}(\mathbf{k})\right)\right)$ with the window level $[-0.5 \mathrm{ppm}, 1 \mathrm{ppm}]$. The bottom row shows the difference image between $\chi_{c}$ in Fig. 2 and $\chi_{\epsilon}$ with the window level [ $\left.-0.5 \mathrm{ppm}, 1 \mathrm{ppm}\right]$. Notice that even for a dipole-compatible field, truncation causes streaking artifacts around the high-frequency regions (interfaces between regions-jump set).

QSM. Let $\chi:\left(\Omega \subset \mathbb{R}^{3}\right) \rightarrow \mathbb{R}$, then MEDI minimizes

$$
E_{\mathrm{MEDI}}(\chi):=\int_{\Omega}|w(d * \chi-b)|^{2}+\lambda \int_{\Omega}|\mathbb{M} \nabla \chi|_{1} .
$$

Here, $w: L^{2}(\Omega) \rightarrow L^{2}(\Omega)$ is the SNR (signal-to-noise ratio) weighting and $\mathbb{M}$ is a matrix acting on $\nabla \chi$. Then, the integrand in the regularization term is defined as

$$
\left.|\mathbb{M}(\mathbf{r}) \nabla \chi(\mathbf{r})|_{1}=\sum_{i=x, y, z} \mid\left(1-\delta_{i}(\mathbf{r})\right) \partial_{i} \chi(\mathbf{r})\right) \mid .
$$

Here, $\delta_{i}(\mathbf{r})$ is an edge indicator for the $i$-direction at $\mathbf{r}$. For example, $\delta_{x}\left(\mathbf{r}_{0}\right)=1$ if there is an edge along the $x$ axis at $\mathbf{r}_{0}$; otherwise $\delta_{x}\left(\mathbf{r}_{0}\right)=0$. These edge indicators are derived from the magnitude image associated with the unknown susceptibility map under the assumption that edges in the two images coexist. Therefore, the weighted anisotropic total variation $\int_{\Omega}|\mathbb{M} \nabla \chi|_{1}$ penalizes only the regions where tissue structure is not expected [93].

Remark 4: The 2-norm is also applicable for the integrand in the regularization term, which is known as weighted isotropic total variation (TV). As opposed to anisotropic TV, 


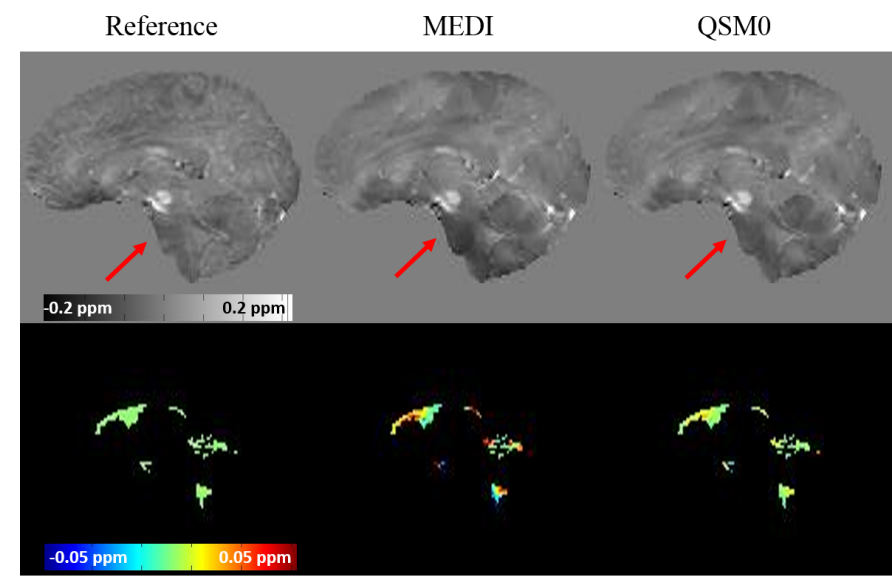

Fig. 6. Simulation result. Top row: Reference QSM (left) which does not contain white matter anisotropy, MEDI (middle) and QSM0 (right) generated from a field which contains white matter anisotropy. Bottom row: Highlighted QSM for CSF. Notice the reduced shadow artifact indicated by the red arrows in QSM0 compared to MEDI. Meanwhile, the homogeneity of CSF is improved using QSM0.

isotropic TV is rotation invariant. However, the difference in accuracy between the two norms in the weighted TV for QSM is very subtle; this invites further investigation.

Incorporating tissue edge information into TV is a notable feature of MEDI [93]. Recall that streaking and tissue boundaries have similar penalties in terms of TV, but streaking only appears along the singular support of the double-cone $\Upsilon$, making it distinct from the tissue edges. Furthermore, SNR weighting in the data fidelity term plays an important role in robust fitting. These have been shown in [1, 88, 94]. Note that the objective in (17) does not truncate data in $\mathrm{k}$-space so that more data is exploited.

\section{B. Variations and Flexibility}

Numerous QSM methods have been proposed in the context of Bayesian image reconstruction with MEDI. These methods can be understood as variations of MEDI-their common goal is to reduce streaking artifacts-and include:

- Nonlinear MEDI 95

- Compressed sensing compensated (CSC) inversion [75]

- Homogeneity enabled incremental dipole inversion (HEIDI) [74]

- Total generalized variation (TGV) based inversion $[96$ 97]

- Iterative LSQR (iLSQR) [98]

- Quantifying susceptibility by inversion of a perturbation (QSIP) model [99]

- Vessel orientation constrained QSM [100]

- Structural featured based collaborative reconstruction (SFCR) [101]

- MEDI based on anisotropic weighting [102]

These methods can be combined in a plug-and-play fashion to tackle targeted applications. For instance, the nonlinear data fidelity term proposed in [95] can be combined with TGV [96. 97| to reduce noise and effects of phase unwrapping failures as

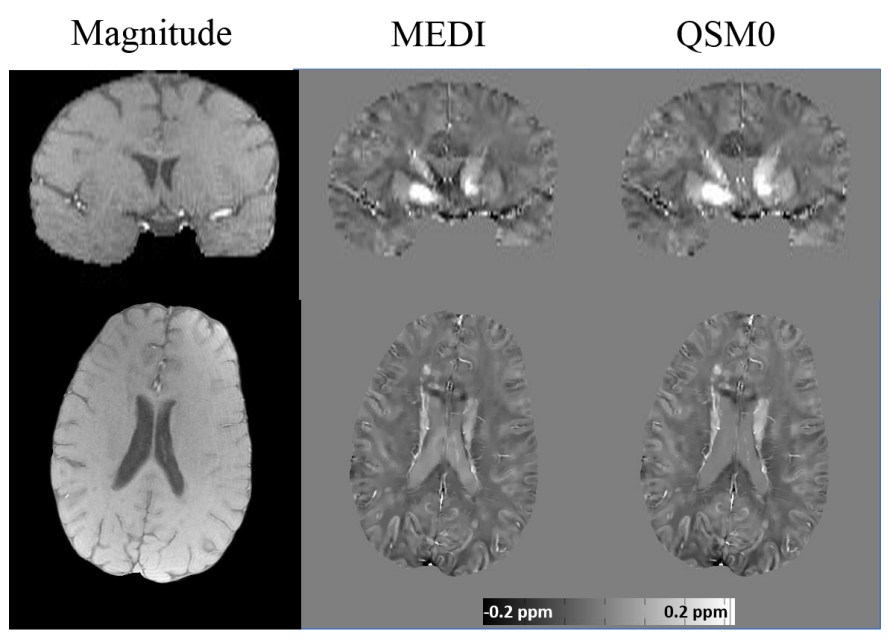

Fig. 7. Comparison between magnitude image (left), QSM reconstructed using MEDI (middle) and QSM0 (right) for in vivo data. Ventricles appear more homogeneous in QSM0 with reduced shadows compared to MEDI. Here, SPURS [104] and PDF [67] were used for phase unwrapping and background field removal, respectively.

well as the undesirable staircasing effect [103], i.e., artificial piecewise constant regions, caused by TV regularization. Two regularization terms can be also combined, e.g., anisotropic weighting [102] and TGV to promote the parallel orientation between the magnitude and susceptibility edges, and to reduce the staircasing effect. The flexibility of the Bayesian MAP estimation framework is a unique and appealing feature in that new priors will continue to be proposed.

\section{Reducing Shadow and zero reference-QSMO}

MEDI and its variations listed above can effectively reduce strong streaking by TV-based regularization. However, they are inherently incapable of penalizing slowly fluctuating artifacts as demonstrated in [88] because the gradient of such fluctuation is much smaller than that of streaking. A naïve approach is to make use of a high pass filter, however this also filters out desired susceptibility variations below the cutoff frequency. Inspired by [2], cerebrospinal fluid (CSF) regularization to enforce a natural zero susceptibility reference needed for absolute quantification has been shown to reduce shadow artifacts [105, 106], with the following cost function

$$
\begin{aligned}
E_{\mathrm{QSM} 0}(\chi):= & \int_{\Omega}|w(d * \chi-b)|^{2} \\
& +\lambda_{1} \int_{\Omega}|\mathrm{M} \nabla \chi|_{1}+\lambda_{2} \int_{\mathrm{CSF}}|\chi-\overline{\chi \mathrm{CSF}}|^{2},
\end{aligned}
$$

where $\lambda_{1}$ and $\lambda_{2}$ are regularization parameters and $\overline{\chi_{\mathrm{CSF}}}$ is the arithmetic mean of $\chi$ inside the CSF region. The rationale for the second regularization term is as follows: CSF is chemically almost pure water so that its susceptibility distribution is expected to be homogeneous (i.e., the variance of $\chi$ in the CSF region is very small), and the CSF region can be automatically segmented. There are often apparent susceptibility variations in the CSF region, because anisotropic white matter near the region contributes to a dipole-incompatible field. Hence, 
imposing CSF homogeneity in the QSM reconstruction can be an effective way of reducing shadow as demonstrated in Figs. 6 and 7

Remark 5: As its name QSMO reveals, E $E_{\mathrm{QSM}}$ was proposed in the context of CSF zero reference [105. 106]. Additionally, it has the benefit of reducing shadow that has low spatial frequency variations over the CSF region. In general, shadow artifacts may be minimized by penalizing such low spatial frequency variations where tissues are expected to be uniform.

\section{Computational Methods-Solvers}

Bayesian QSM methods need to be solved by iterative methods because writing out an explicit solution for the EulerLagrange equation associated with the objective is difficult or impossible. Here, we focus on minimizing $E_{\mathrm{MEDI}}$ in (17). The major algorithmic challenge in this optimization comes from the presence of TV which is nonsmooth (albeit convex). We present three well-established algorithms for MEDI which can be also applied to the variations listed in Section IV-B. Quasi-Newton, split-Bregman, and primal-dual algorithms.

We start with a standard discrete setting for minimizing (17). As discussed in [107], an improper discretization scheme for the differential operator in (17) leads to inaccuracy as well as visual artifacts in the final susceptibility map. Keeping this in mind, consider a $3 \mathrm{D}$ regular Cartesian grid of size $N_{x} \times N_{y} \times$ $N_{z}$ as follows:

$$
\left\{\left(i h_{x}, j h_{y}, k h_{z}\right): 1 \leq i \leq N_{x}, 1 \leq j \leq N_{y}, 1 \leq k \leq N_{z}\right\},
$$

where $h_{x}, h_{y}$, and $h_{z}$ denote the voxel size and $(i, j, k)$ denotes the voxel location. Then, $\chi$ is discretized in $\mathcal{H}=\mathbb{R}^{N_{x} \times N_{y} \times N_{z}}$ where a scalar product is defined as

$$
\langle\chi, \psi\rangle_{\mathcal{H}}=\sum_{i, j, k} \chi_{i, j, k} \psi_{i, j, k}, \quad \chi, \psi \in \mathcal{H} .
$$

For $\chi \in \mathcal{H}$, the discrete gradient is defined by

$$
(\nabla \chi)_{i, j, k}=\left((\nabla \chi)_{i, j, k}^{x},(\nabla \chi)_{i, j, k}^{y},(\nabla \chi)_{i, j, k}^{z}\right),
$$

where

$$
\begin{aligned}
& (\nabla \chi)_{i, j, k}^{x}= \begin{cases}\left(\chi_{i+1, j, k}-\chi_{i, j, k}\right) / h_{x} & \text { if } i<N_{x}, \\
0 & \text { if } i=N_{x}\end{cases} \\
& (\nabla \chi)_{i, j, k}^{y}= \begin{cases}\left(\chi_{i, j+1, k}-\chi_{i, j, k}\right) / h_{y} & \text { if } j<N_{y}, \\
0 & \text { if } j=N_{y}\end{cases} \\
& (\nabla \chi)_{i, j, k}^{z}= \begin{cases}\left(\chi_{i, j, k+1}-\chi_{i, j, k}\right) / h_{z} & \text { if } k<N_{z}, \\
0 & \text { if } k=N_{z} .\end{cases}
\end{aligned}
$$

Notice that the forward difference scheme with the Neumann boundary condition is used for the discrete gradient [108, 109]. Also, the gradient vector $\nabla \chi$ is a vector in $\mathcal{K}=\mathcal{H} \times \mathcal{H} \times \mathcal{H}$ where a scalar product is given as

$\langle\xi, \zeta\rangle_{\mathcal{K}}=\sum_{i, j, k} \xi_{i, j, k}^{x} \zeta_{i, j, k}^{x}+\xi_{i, j, k}^{y} \zeta_{i, j, k}^{y}+\xi_{i, j, k}^{z} \zeta_{i, j, k}^{z}, \quad \xi, \zeta \in \mathcal{K}$.

Now, we define a linear map $\mathbb{M}: \mathcal{K} \rightarrow \mathcal{K}$ such that

$$
\xi_{i, j, k} \mapsto(\mathbb{M} \xi)_{i, j, k}=\left(\mathbb{M}_{i, j, k}^{x} \xi_{i, j, k}^{x}, \mathbb{M}_{i, j, k}^{y} \xi_{i, j, k}^{y}, \mathbb{M}_{i, j, k}^{z} \xi_{i, j, k}^{z}\right)
$$

The objective in (17) is discretized accordingly, yielding the following optimization problem in a finite dimensional vector space:

$$
\hat{\chi} \in \operatorname{argmin}_{\chi}\|w(d * \chi-b)\|_{2}^{2}+\lambda\|\mathbb{M} \nabla \chi\|_{1,1},
$$

where $w: \mathcal{H} \rightarrow \mathcal{H}$ is SNR weighting, $d$ is the discretized dipole kernel, and $b \in \mathcal{H}$ is the RDF. We define $\|\cdot\|_{1,1}$ as

$$
\|\xi\|_{1,1}=\sum_{i, j, k}\left|\xi_{i, j, k}^{x}\right|+\left|\xi_{i, j, k}^{y}\right|+\left|\xi_{i, j, k}^{z}\right| .
$$

After vectorization, the formulation (18) can be rewritten as:

$$
\hat{\chi} \in \operatorname{argmin}_{\chi}\left\|W\left(F^{H} D F \chi-b\right)\right\|_{2}^{2}+\lambda\|M G \chi\|_{1}
$$

where $W$ is a diagonal matrix associated with $w, F$ is the discrete Fourier transform (DFT) matrix, $D$ is a diagonal matrix whose diagonal elements correspond to the Fourier transform of $d$, and the superscript $H$ denotes the Hermitian transpose in the data fidelity term. The finite difference matrix $G: \mathbb{R}^{N_{x} N_{y} N_{z}} \rightarrow \mathbb{R}^{3 N_{x} N_{y} N_{z}}$ is constructed from the forward difference scheme with the Neumann boundary condition, and $M$ is a diagonal matrix associated with $\mathbb{M}$.

\section{A. Quasi-Newton Method (Gauss-Newton in QSM)}

Since the optimization problem (19) is convex, a necessary and sufficient condition for optimality of $(19)$ is to find $\hat{\chi}$ such that $0 \in \partial_{\chi} E(\hat{\chi})$, where $E(\chi):=\left\|W\left(F^{H} D F \chi-b\right)\right\|_{2}^{2}+$ $\lambda\|M G \chi\|_{1}$. Here, $\partial_{\chi} E(\chi)$ is given as

$$
\begin{aligned}
\partial_{\chi} E(\chi)= & 2\left(W F^{H} D F\right)^{H}\left(W F^{H} D F\right) \chi-\left(W F^{H} D F\right)^{H} b \\
& +\lambda(M G)^{H}\left(\frac{M G \chi}{\sqrt{\operatorname{diag}(M G \chi)^{2}}}\right) .
\end{aligned}
$$

Then, the lagged diffusivity fixed-point iteration (LDFPI) method [110, 111] is used to linearize the nonlinear equation $\partial_{\chi} E(\chi)=0$ by substituting $1 / \sqrt{\operatorname{diag}(M G \chi)^{2}}$ with $1 / \sqrt{\operatorname{diag}\left(M G \chi^{k}\right)^{2}+\epsilon}$, where $\chi^{k}$ denotes the $k$-th iterate and $\epsilon$ is a strictly positive relaxation parameter which avoids division by zero. Note that $\partial_{\chi}\|M G \chi\|_{1}$ leads to a nonlinear diffusion equation, and this linearization procedure makes the diffusivity coefficient lag one step (iteration) behind. LDFPI then computes $\chi^{k+1}$ by solving the following linear system

$$
L\left(\chi^{k}\right) \chi^{k+1}=\tilde{b},
$$

where $\quad L\left(\chi^{k}\right) \quad:=\quad 2\left(W F^{H} D F\right)^{H}\left(W F^{H} D F\right) \chi+$ $\lambda(M G)^{H}(M G) / \sqrt{\operatorname{diag}\left(M G \chi^{k}\right)^{2}+\epsilon}, \quad$ and $\quad \tilde{b} \quad:=$ $\left(W F^{H} D F\right)^{H} b$. Once $\chi^{k+1}$ is computed, $\chi^{k} \leftarrow \chi^{k+1}$ and solve (21) until $\left\|\chi^{k+1}-\chi^{k}\right\|$ becomes sufficiently small (fixed-point iteration). The rate of convergence largely depends on how fast LDFPI solves a series of the linear systems 21. Krylov subspace methods such as conjugate gradient $(\mathrm{CG})$ can be used with preconditioning techniques [112

From (20) and 21], observe that $\tilde{b}=L\left(\chi^{k}\right) \chi^{k}-\partial_{\chi} E\left(\chi^{k}\right)$. Using this relation, we can rewrite (21) as follows:

$$
L\left(\chi^{k}\right) \delta \chi=-\partial_{\chi} E\left(\chi^{k}\right),
$$




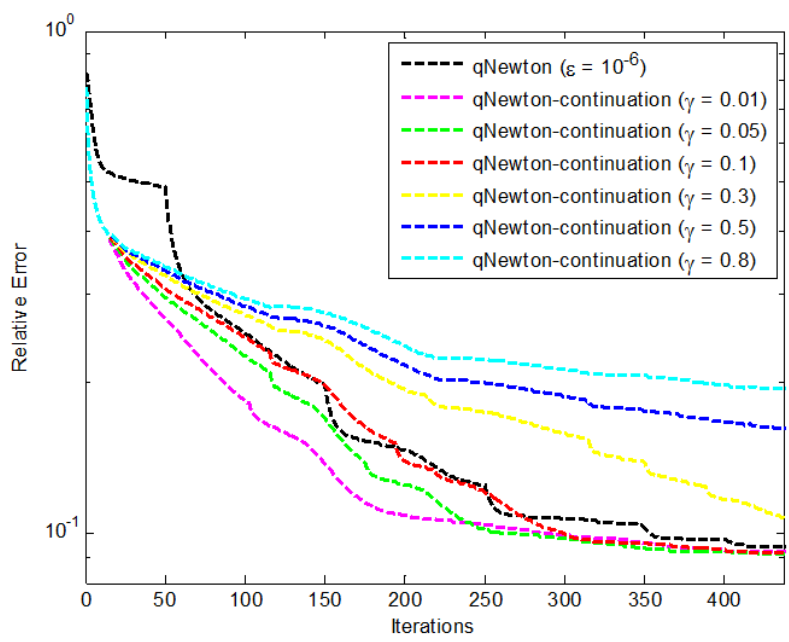

Fig. 8. The continuation method helps speed up the quasi-Newton formulation of LDFPI (qNewton) if the decreasing factor $\gamma$ is properly chosen (see the purple, green, and red curves below the black). Continuation converges rather slowly when $\gamma \geq 0.3$; see the yellow, blue, and cyan curves above the black.

where $\delta \chi=\chi^{k+1}-\chi^{k}$. Then, solving (22) with respect to $\delta \chi$ is a quasi-Newton method where $L\left(\chi^{k}\right)$ is an estimate of the Hessian matrix of $E(\chi)$ at $\chi^{k}$. To see this, consider the second order Taylor expansion of $E(\chi)$ around $\chi^{k}$ as follows:

$E\left(\chi^{k}+\delta \chi\right) \simeq E\left(\chi^{k}\right)+\partial_{\chi} E\left(\chi^{k}\right)^{\top} \delta \chi+\frac{1}{2} \delta \chi^{\top}\left[H E\left(\chi^{k}\right)\right] \delta \chi$,

where $H E\left(\chi^{k}\right)$ is the Hessian matrix. Newton's method sets the derivative of this expansion with respect to $\delta \chi$ equal to zero:

$$
H E\left(\chi^{k}\right) \delta \chi=-\partial_{\chi} E\left(\chi^{k}\right)
$$

where

$$
\begin{aligned}
& H E\left(\chi^{k}\right) \\
& =L\left(\chi^{k}\right)+\left.\lambda(M G)^{H}\left(M G \chi^{k}\right) \partial_{\chi}\left(\frac{1}{\sqrt{\operatorname{diag}(M G \chi)^{2}+\epsilon}}\right)\right|_{\chi=\chi^{k}} .
\end{aligned}
$$

$L\left(\chi^{k}\right)$ is an estimate of the Hessian $H E\left(\chi^{k}\right)$ by dropping the last term from 23. The quasi-Newton approach, therefore, iteratively approximates the original nonlinear minimization problem 19 by a series of quadratic minimization problems. An advantage of the quasi-Newton form (22) over the fixedpoint form (21) is that 22) tends to be less sensitive to roundoff error 113 .

The accuracy of the quasi-Newton form (22) depends on the value of the numerical relaxation parameter $\epsilon$, which, by default, is set to $10^{-6}$ for QSM. Accuracy can be improved by decreasing $\epsilon$ but this comes at the cost of significantly slowing down the rate of convergence [107]. This can be overcome by using the so-called continuation method. That is, at each outer iteration, we reset $\epsilon$ by the rule $\epsilon \leftarrow \gamma \cdot \epsilon$ where $\gamma$ is a decreasing factor. We initialize $\epsilon=0.1$ and set $\gamma$ to values ranging from 0 to 1 . Fig. 8 shows relative errors when $\gamma$ is $0.01,0.05,0.1,0.3,0.5$, and 0.8 .
Remark 6: This quasi-Newton method has been frequently referred to as Gauss-Newton in the QSM literature [1]. This may be because Newton's method has been often referred to as the Newton-Raphson method for finding roots in physics.

\section{B. Split-Bregman Method}

The split-Bregman method [114] makes use of variable splitting and the Bregman iteration. Unlike the quasi-Newton method, this solves (19) without numerical approximation for TV regularization. The method of variable splitting reformulates (19) as

(Constrained)

$=\operatorname{argmin}_{(\chi, v)}\left\|W\left(F^{H} D F \chi-b\right)\right\|_{2}^{2}+\lambda\|v\|_{1}$ s.t. $v=M G \chi$.

A quadratic penalty is incorporated and we have

(Unconstrained)

$=\operatorname{argmin}_{(\chi, v)}\left\|W\left(F^{H} D F \chi-b\right)\right\|_{2}^{2}+\lambda\|v\|_{1}+\frac{\rho}{2}\|v-M G \chi\|_{2}^{2}$.

This can be solved by alternating continuation, i.e., taking $\rho \rightarrow \infty$ one alternatively minimizes the objective with respect to $\chi$ and $v$. However, the problem becomes ill-conditioned as $\rho \rightarrow \infty$ [115]. Instead of taking $\rho \rightarrow \infty$, the Bregman iteration [116] is used with a fixed value of $\rho$. This procedure makes its numerical behavior much more stable; the iterative sequence is as follows:

$$
\begin{aligned}
& \chi^{k+1} \\
& =\operatorname{argmin}_{\chi}\left\|W\left(F^{H} D F \chi-b\right)\right\|_{2}^{2}+\frac{\rho}{2}\left\|v^{k}-M G \chi-z^{k}\right\|_{2}^{2} \\
& =\operatorname{argmin}_{\chi}\left\|\left[\begin{array}{c}
W F^{H} D F \\
\sqrt{\rho / 2} M G
\end{array}\right] \chi-\left[\begin{array}{c}
W b \\
\sqrt{\rho / 2}\left(v^{k}-z^{k}\right)
\end{array}\right]\right\|_{2}^{2} \\
& v^{k+1} \\
& =\operatorname{argmin}_{v} \lambda\|v\|_{1}+\frac{\rho}{2}\left\|v-M G \chi^{k+1}-z^{k}\right\|_{2}^{2} \\
& =\operatorname{prox}_{\rho / \lambda,\|\cdot\|_{1}}\left(M G \chi^{k+1}+z^{k}\right) \\
& z^{k+1} \\
& =z^{k}+\left(M G \chi^{k+1}-v^{k+1}\right)
\end{aligned}
$$

where Krylov subspace methods, e.g., CG or LSQR, can be used for (24). The proximal operator $\operatorname{prox}_{\rho / \lambda,\|\cdot\|_{1}}(\cdot)$ in 25] is given as soft-thresholding [115]. The last line of the algorithm (26) is known as the Bregman update and its derivation can be found in [116]. In QSM, the split-Bregman method was applied to [19] without SNR weighting in [117] to accelerate the speed of deconvolution. The method was subsequently considered with SNR weighting in [118].

Remark 7: Note that the split-Bregman method with linear constraints is equivalent to the alternating direction method of multipliers (ADMM) [115]. Hence, its convergence can be analyzed by $A D M M$.

\section{Primal-Dual Algorithm}

Similar to the distributional derivative for a function whose differentiation is not pointwise defined [83, 119], we 
can derive a generalized (dual) definition of the weighted (anisotropic) TV [107, 120] as follows:

$$
\int_{\Omega}|\mathbb{M} \nabla \chi|_{1}=\sup _{\xi:\|\xi\|_{\infty} \leq 1} \int_{\Omega}\langle\mathbb{M} \nabla \chi, \xi\rangle,
$$

where the dual variable $\xi: \Omega \rightarrow \mathbb{R}^{3}$ is a smooth map and $\|\cdot\|_{\infty}$ is the pointwise maximum norm. Replacing the TV term in $E_{\text {MEDI }}$ with its dual formulation (27), we notice that minimizing $E_{\mathrm{MEDI}}$ in $\chi$ is equivalent to finding a saddle-point $(\hat{\chi}, \hat{\xi})$ such that

$$
\min _{\chi} \max _{\xi:\|\xi\|_{\infty} \leq 1} \int_{\Omega}|w(d * \chi-b)|^{2}+\lambda \int_{\Omega}\langle\mathbb{M} \nabla \chi, \xi\rangle .
$$

Then, one can use gradient descent in $\chi$ and gradient ascent in $\xi$ with the relation $\langle\mathbb{M} \nabla \chi, \xi\rangle=\left\langle\chi,(\mathbb{M} \nabla)^{H} \xi\right\rangle$. The data term can be further dualized to avoid solving a linear system that appears as a nested routine as seen in the quasi-Newton and split-Bregman methods. For technical and implementation details, we refer the reader to [107].

\section{Final Remarks on Solvers}

As opposed to Newton's method in optimization that makes use of the Hessian matrix of the objective function, first-order methods (quasi-Newton, split-Bregman, and primal-dual) have a linear rate of convergence for the complete class of nonsmooth convex problems [121]. Since only subtle differences in speed and accuracy are observed, any method can be used, for instance NESTA [122] can be also used. That being said, it is unclear if second-order methods (Newton-type methods) would outperform the first-order methods because they require computation of the Hessian matrix at each step; this is not only expensive, but also noise sensitive [123].

New regularization terms may appear nonconvex, therefore the first-order methods may not be useful in finding a nearoptimal solution. In this case, the Hessian matrix can help escape saddle points towards a globally optimal solution [124]. Algorithmic advances will be then necessary to catch up with realistic nonconvex energies.

\section{Vi. Computational Methods-Preconditioning}

Numerical computation of a solution in Bayesian QSM can be accelerated using preconditioning techniques that incorporate prior knowledge of the targeted susceptibility solution. As known in matrix inversion [125, 126], a well-chosen left/right preconditioner can accelerate the rate of convergence of an iterative solver. In Bayesian MAP estimation, preconditioning may play a role in guiding the path of a minimizing sequence in such a way that it emphasizes certain properties of a final solution [127, 128]. Here, we present an overview of recent advances in background field removal methods in the context of preconditioning.

\section{A. Background Field Removal}

The field generated by the sources inside a tissue volume of interest (VOI) such as the brain, known as tissue (local) field, turns out to be much smaller than the field generated by the sources outside the VOI, which include strong susceptibility gradients at air-tissue interfaces and in the skull, known as background field [129]. A typical QSM strategy is to first perform data fitting and removal of this background field on the total field estimated from MRI phase, which is referred to as background field removal [1, 2, 67, 78]. The tissue field after background field removal is then inverted to generate a tissue susceptibility map. Because background field removal does not require precise susceptibility source localization, it can be executed rapidly. With the removal of the background field, the susceptibility range to be searched for mapping tissue susceptibility can be reduced by $\sim 10$ fold (but this breaks down in case of hemorrhage [95, 130], leading to a rapid convergence in solving (17). Therefore, current QSM algorithms typically consist of two steps [1]: background field removal and tissue field inversion.

As discussed in [1], the lack of MRI signal in the background is one of the QSM challenges to determine the background field inside the VOI accurately. All current methods to remove the background field in the tissue VOI are based on Maxwell's equations, which state that the background field in the tissue VOI obeys the homogeneous Laplacian equation. The lack of boundary condition makes it an ill-posed problem to solve this Laplacian equation [129]. Numerous formulations of prior knowledge have been proposed for estimating background field, including:

- High pass filtering (HPF) [131-133]

- Projection onto dipole fields (PDF) 67

- Sophisticated harmonic artifact reduction for phase data (SHARP) [62]

- Laplacian boundary value (LBV) method [129]

- Iterative spherical mean value method (iSMV) [134]

- Regularization-enabled SHARP (RESHARP) [135]

For a complete review on background field removal methods, we refer the reader to [78]. It should be noted that these prior knowledge formulations contain error, and the background field removal assumption of reducing the susceptibility search range fails when susceptibility values are large, such as in the case of hemorrhage [130]. It was recently shown that a tissue susceptibility map could be recovered without background field removal but this required a long processing time [130], because a tissue susceptibility map was still computed from the total field by minimizing $E_{\mathrm{MEDI}}$ in 17 . This work elucidates the rate of convergence of an iterative solver, demonstrating that the background field removal step plays a role in preconditioning in a classical sense. It also demonstrates that the error in a background field removal method can be avoided by focusing on minimizing $E_{\mathrm{MEDI}}$ from the total field.

The remaining concern for minimizing $E_{\mathrm{MEDI}}$ is systematically incorporating preconditioning in such a way that its solution is computed as fast as the current two-step procedure (background field removal + tissue field inversion) in Bayesian QSM. In the following sections, we present two notable examples.

\section{B. Preconditioned MEDI}

Preconditioning can be systematically incorporated into a Bayesian QSM method [130] by modifying the cost function 
(a)

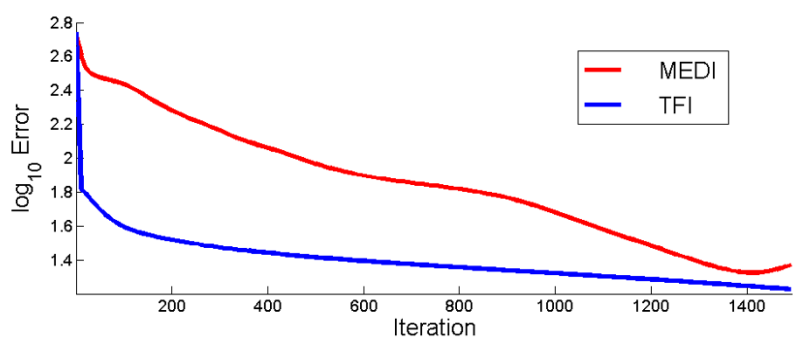

(b)

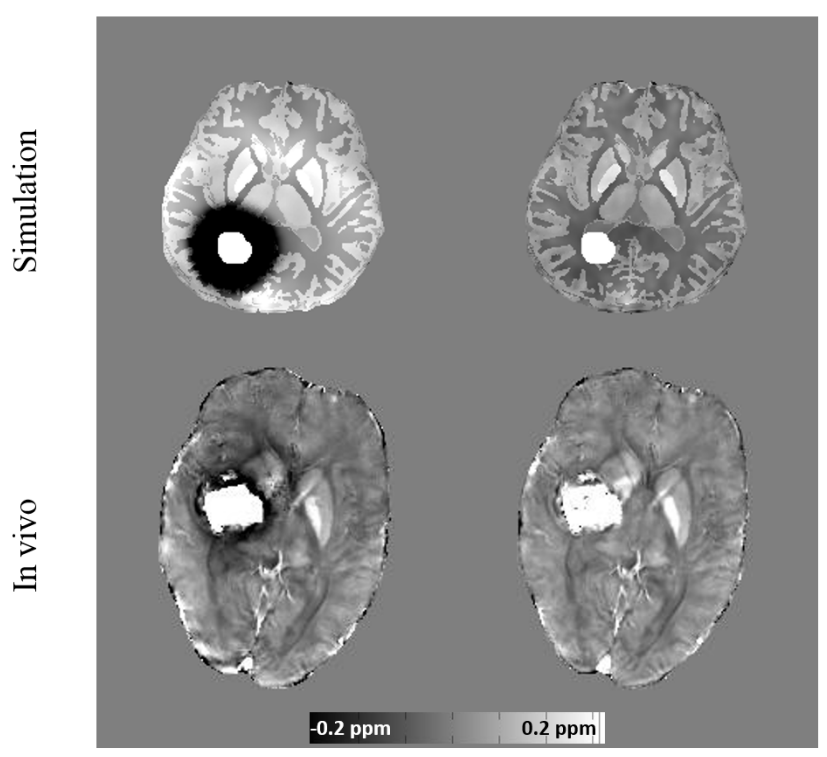

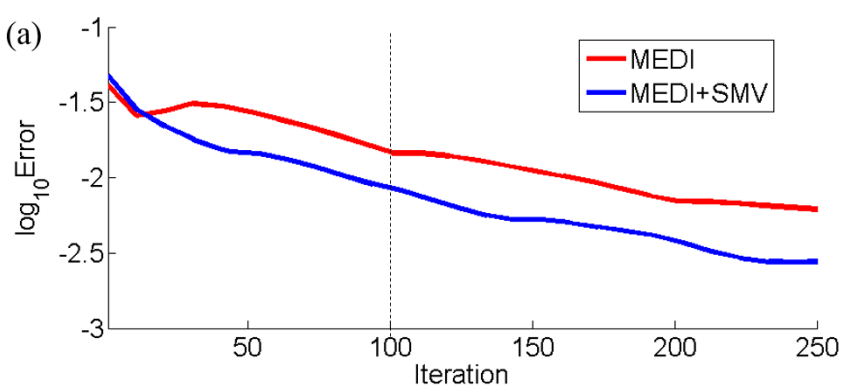

(b)

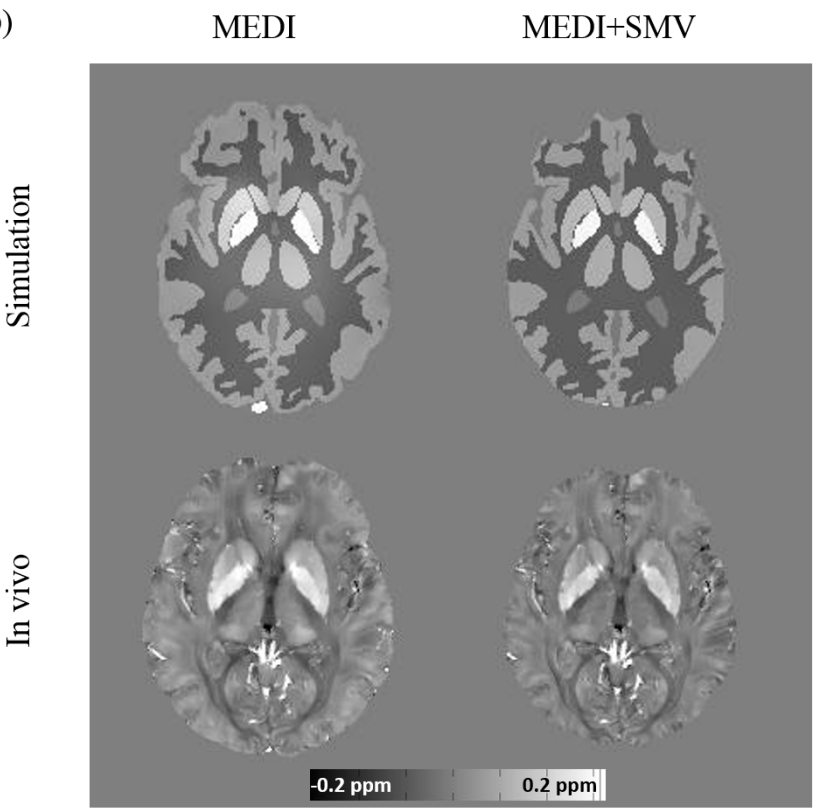

Fig. 9. MEDI versus preconditioned MEDI (TFI). (a) Plot of error between estimated and true brain susceptibility with respect to the CG iteration number in numerical simulation (b) QSM reconstructed by MEDI and TFI in numerical simulation and in vivo. Notice that preconditioning dramatically reduces the hypointense artifact around the hemorrhage site. For both MEDI and TFI, SPURS [104] was used for phase unwrapping, and PDF [67] was additionally used for background field removal for MEDI.

$E_{\text {MEDI }}$ in (17) with a preconditioner $P$ :

$$
E_{\mathrm{PMEDI}}(P y):=\int_{\Omega}|w(d * P y-b)|^{2}+\lambda \int_{\Omega}|\mathbb{M} \nabla P y|_{1} .
$$

which is minimized with respect to $y$ to generate a susceptibility solution $\chi=P y$. Here, $b$ is the total field, and the preconditioner $P$ is chosen from prior knowledge about the distribution of brain susceptibility. In the case of QSM reconstruction of a patient with intracerebral hemorrhage, $P$ is chosen as a binary matrix with higher weights in the hemorrhage than other regions to reflect the strong susceptibility contrast between the hemorrhage and the surrounding brain tissue [130]. Both simulation and in vivo results (Fig. 9] show that the preconditioner helps suppress shadow artifacts adjacent to the hemorrhage site by improving the convergence behavior of CG involved in the inner loop of the quasi-Newton method presented in Section $\mathrm{V}-\mathrm{A}$

\section{Differential Model}

Along with the integral approach in 177 where the data term is derived from (8), differential approaches proposed in [136-
Fig. 10. Comparison between MEDI and MEDI + SMV. (a) Plot of error between estimated and true brain susceptibility with respect to the CG iteration number in numerical simulation. (b) QSM reconstructed by MEDI and MEDI + SMV in numerical simulation (100 CG iterations) and in vivo (150 CG iterations). MEDI + SMV produces more homogeneous map at the cost of brain erosion. Here, SPURS [104 and PDF [67 were used for phase unwrapping and background field removal, respectively.

[139] are also commonly used Bayesian QSM methods which can be understood in the context of preconditioning. These approaches make use of the PDE (4) or (9) as the data fidelity term of $E_{\mathrm{MEDI}}$ in (17). As a result, a background field removal step is no longer needed, making these approaches as fast as the two-step (e.g., PDF + MEDI) procedure. Additionally, it has been shown that some of the approaches known as singlestep QSM [138, 139] have less shadow artifacts. Recall that the integral form (8) is derived from the differential form (4) with the assumption that the Fourier transform of each term in (4) exists and $\mathbf{k} \neq \mathbf{0}$. Therefore, both integral and differential representations appear to be equivalent. Yet, a rigorous analysis needs to be carried out because the differential approach seems more capable of reducing shadow artifacts than the integral approach.

From a computational point of view, this behavior may be explained by the $4 \pi|\mathbf{k}|^{2}$ left preconditioner that comes from the Laplacian operator. In other words, as opposed to the data term of the integral approach in (17), the following differential 
term

$$
\int_{\Omega}|\tilde{w} \Delta(d * \chi-b)|^{2}=\int_{\Omega}\left|\tilde{w} \mathcal{F}^{-1}\left[4 \pi|\mathbf{k}|^{2}(D X-B)\right]\right|^{2}
$$

may emphasize the high frequency components of a candidate susceptibility distribution more than its low frequency components containing slowly varying dark shadows. Note that unlike the single-step QSM model [138, 139], here we have considered the SNR weighting $\tilde{w}$ which is modified from $w$ in the integral approach (17) as follows: Let $L$ be the Laplacian operator in a matrix form, and let $n$ be the random noise vector $n=d * \chi-b$. Since we define $w$ as the inverse of the square root of the covariance matrix of the noise vector $n$, a proper way of determining the new SNR weighting $\tilde{w}$ is to set it to the inverse of the square root of the covariance matrix associated with $L n$.

The differential approach can be implemented by using the spherical mean value (SMV) kernel and incorporated into $E_{\mathrm{MEDI}}$; this is denoted by MEDI + SMV. Here, we use the diagonal components of the inverse of the square root of the covariance matrix associated with $L n$ for $\tilde{w}$. Fig. 10 shows both simulation and in vivo results, where the tissue field contains unresolved background fields and is used as an input for both MEDI and MEDI + SMV. This SMV kernel considerably improves the rate of convergence of CG. Visually, MEDI + SMV produces a more homogeneous map than MEDI. However, any SMV implementation causes erosion of the VOI region since the field outside the VOI cannot be measured. This causes loss of anatomical information at the boundaries of the brain, especially when the voxel size is large.

Remark 8: These Bayesian preconditioning techniques have only been empirically explored. A rigorous analysis is required as preconditioning seems to give promising results in QSM.

\section{DISCUSSION AND CONCLUSION}

QSM based on the Bayesian MAP estimation framework can accurately and robustly map strong isotropic sources, such as biometals including iron in ferritin and deoxy-heme in hemoglobin [8, 70], calcium in bones and other pathologic calcifications [43, 48], and highly paramagnetic gadolinium and iron in contrast agents [49, 50]. The accuracy and robustness of Bayesian MAP estimation are well established, such as in image processing known to outperform classical multistep algorithms for tasks including denoising [140], deblurring [141], segmentation [142], and optical flow estimation [143]. Effective QSM applications in clinical problems that are often complex require further technical innovation. Although we have used the convex cost in (17) for elucidating the Bayesian QSM framework, more sophisticated priors capable of precisely modeling the underlying susceptibility distribution are desired so that streaking and shadow can be better penalized. Fast and robust algorithms that can handle nonconvex priors are often necessary as such priors may well be no longer convex, such as when fat presents in tissue [48, 104, 144, 145] or when SNR is poor [95]. As seen in Section VI, Bayesian methods benefit from further investigation in preconditioning techniques. These innovations may be made based on a deeper understanding of the biophysics behind tissue magnetic susceptibility and solvers in numerical optimization.

A particularly complex problem is the molecular microstructure and its anisotropic susceptibility of white matter (WM) in the brain [146]. This WM susceptibility anisotropy has been attributed to anisotropic lipid molecules that are radially oriented in the myelin sheathes of white matter fibers [71, 147-149]. Accordingly, a hollow cylinder fiber model may account for effects from both microstructure and anisotropy of WM [150], which is used to study the orientation of WM fibers [151-153]. WM microstructure can be inspected from the orientation dependent local frequency shift [154, 155]. The molecular anisotropy of WM in a non-local frequency perturbation [156] may be estimated using various inversion algorithms [61, 71, 147, 148]. A recent and more complex model combines the generalized Lorentzian approach [154] with the lipoprotein structure of myelin sheath [157] and water attempting to explain experimental phase data [158]. Although QSM can be used to study WM fibers using multiorientation scans, a reliable measurement of anisotropy in WM and microstructure in a voxel from a single-angle acquisition has not been achieved. In fact, clinical MR scanners do not allow "perfect" multi-orientation sampling using a combination of uniformly distributed field directions because of the limited range of orientations with human subjects [148].

In summary, we have tried to provide a systematic overview of the current status and recent advances in QSM methods, and remaining challenges. The notion of a fundamental solution in the theory of linear PDEs makes it possible to write out an explicit form of the solution for the magnetic field to susceptibility source inversion problem. We have demonstrated that streaking and shadows are two types of artifacts characterized by the PDE solution for field data deviating from or incompatible with the continuous dipole field pattern. As dipole-incompatibility in field data is unavoidable in practice, it is critical to devise effective methods capable of reducing streaking and shadow artifacts. Early attempts that make use of truncation in k-space unnecessarily cause dipoleincompatibility in the field, giving rise to additional streaking and shadows. On the other hand, Bayesian QSM methods provide desired results with significant improvements over the truncation-based methods, allowing for the design of problemspecific and physics-based priors. The QSM community continues to devise new priors that will improve the current state of the art. As these new priors may be nonconvex, they will be more capable of modeling the complicated biophysical nature behind QSM; therefore, the community looks forward to algorithmic advances with preconditioning techniques.

\section{ACKNOWLEDGMENT}

This work was supported in part by grants from National Institute of Health (R01 NS095562, R01 NS090464, R01 NS072370, S10 OD021782). Jin Keun Seo was supported by NRF grant 2015R1A5A1009350.

\section{REFERENCES}

[1] Y. Wang and T. Liu, "Quantitative susceptibility mapping (qsm): decoding mri data for a tissue magnetic biomarker," Magnetic resonance in medicine, vol. 73, no. 1, pp. 82-101, 2015. 
[2] L. de Rochefort, T. Liu, B. Kressler, J. Liu, P. Spincemaille, V. Lebon, J. Wu, and Y. Wang, "Quantitative susceptibility map reconstruction from $\mathrm{mr}$ phase data using bayesian regularization: validation and application to brain imaging," Magnetic Resonance in Medicine, vol. 63, no. 1, pp. 194-206, 2010.

[3] M. A. Bernstein, K. F. King, and X. J. Zhou, Handbook of MRI pulse sequences. Elsevier, 2004

[4] E. M. Haacke, S. Liu, S. Buch, W. Zheng, D. Wu, and Y. Ye, "Quantitative susceptibility mapping: current status and future directions," Magnetic resonance imaging, vol. 33, no. 1, pp. 1-25, 2015.

[5] J. Reichenbach, F. Schweser, B. Serres, and A. Deistung, "Quantitative susceptibility mapping: concepts and applications," Clinical neuroradiology, vol. 25, no. 2, pp. 225-230, 2015.

[6] C. Liu, H. Wei, N.-J. Gong, M. Cronin, R. Dibb, and K. Decker, "Quantitative susceptibility mapping: contrast mechanisms and clinical applications," Tomography: a journal for imaging research, vol. 1, no. 1, p. 3, 2015.

[7] S. Eskreis-Winkler, Y. Zhang, J. Zhang, Z. Liu, A. Dimov, A. Gupta, and Y. Wang, "The clinical utility of qsm: disease diagnosis, medical management, and surgical planning," NMR in Biomedicine, vol. 30, no. 4, 2017.

[8] Y. Wang, P. Spincemaille, Z. Liu, A. Dimov, K. Deh, J. Li, Y. Zhang, Y. Yao, K. M. Gillen, A. H. Wilman, A. Gupta, A. J. Tsiouris, I. Kovanlikaya, G. C.-Y. Chiang, J. W. Weinsaft, L. Tanenbaum, W. Chen, W. Zhu, S. Chang, M. Lou, B. H. Kopell, M. G. Kaplitt, D. Devos, T. Hirai, X. Huang, Y. Korogi, A. Shtilbans, G.-H. Jahng, D. Pelletier, S. A. Gauthier, D. Pitt, A. I. Bush, G. M. Brittenham, and M. R. Prince, "Clinical quantitative susceptibility mapping (qsm): Biometal imaging and its emerging roles in patient care," Journal of Magnetic Resonance Imaging, 2017.

[9] A. Deistung, F. Schweser, and J. R. Reichenbach, "Overview of quantitative susceptibility mapping," NMR in Biomedicine, vol. 30, no. 4, 2017.

[10] C. Wisnieff, S. Ramanan, J. Olesik, S. Gauthier, Y. Wang, and D. Pitt, "Quantitative susceptibility mapping (qsm) of white matter multiple sclerosis lesions: interpreting positive susceptibility and the presence of iron," Magnetic resonance in medicine, vol. 74, no. 2, pp. 564-570, 2015.

[11] W. Chen, S. A. Gauthier, A. Gupta, J. Comunale, T. Liu, S. Wang, M. Pei, D. Pitt, and Y. Wang, "Quantitative susceptibility mapping of multiple sclerosis lesions at various ages," Radiology, vol. 271, no. 1, pp. 183-192, 2014.

[12] D. M. Harrison, X. Li, H. Liu, C. K. Jones, B. Caffo, P. A. Calabresi, and $\mathrm{P}$. van Zijl, "Lesion heterogeneity on high-field susceptibility mri is associated with multiple sclerosis severity," American Journal of Neuroradiology, vol. 37, no. 8, pp. 1447-1453, 2016.

[13] X. Li, D. M. Harrison, H. Liu, C. K. Jones, J. Oh, P. A. Calabresi, and P. van Zijl, "Magnetic susceptibility contrast variations in multiple sclerosis lesions," Journal of Magnetic Resonance Imaging, vol. 43, no. 2, pp. 463-473, 2016.

[14] Y. Zhang, S. Gauthier, A. Gupta, W. Chen, J. Comunale, G.-Y. Chiang, D. Zhou, G. Askin, W. Zhu, D. Pitt, and Y. Wang, "Quantitative susceptibility mapping and $\mathrm{r} 2 *$ measured changes during white matter lesion development in multiple sclerosis: myelin breakdown, myelin debris degradation and removal, and iron accumulation," American Journal of Neuroradiology, vol. 37, no. 9, pp. 1629-1635, 2016

[15] Y. Zhang, S. A. Gauthier, A. Gupta, J. Comunale, G. Chia-Yi Chiang, D. Zhou, W. Chen, A. E. Giambrone, W. Zhu, and Y. Wang, "Longitudinal change in magnetic susceptibility of new enhanced multiple sclerosis (ms) lesions measured on serial quantitative susceptibility mapping (qsm)," Journal of Magnetic Resonance Imaging, vol. 44, no. 2, pp. 426-432, 2016.

[16] Y. Zhang, S. Gauthier, A. Gupta, L. Tu, J. Comunale, G.-Y. Chiang, W. Chen, C. Salustri, W. Zhu, and Y. Wang, "Magnetic susceptibility from quantitative susceptibility mapping can differentiate new enhancing from nonenhancing multiple sclerosis lesions without gadolinium injection," American Journal of Neuroradiology, vol. 37, no. 10, pp. 1794-1799, 2016.

[17] S. Eskreis-Winkler, K. Deh, A. Gupta, T. Liu, C. Wisnieff, M. Jin, S. A. Gauthier, Y. Wang, and P. Spincemaille, "Multiple sclerosis lesion geometry in quantitative susceptibility mapping (qsm) and phase imaging," Journal of Magnetic Resonance Imaging, vol. 42, no. 1, pp. 224-229, 2015.

[18] J. Acosta-Cabronero, G. B. Williams, A. Cardenas-Blanco, R. J. Arnold, V. Lupson, and P. J. Nestor, "In vivo quantitative susceptibility mapping (qsm) in alzheimer's disease," PloS one, vol. 8, no. 11, p. e81093, 2013.
[19] E.-J. Hwang, H.-G. Kim, D. Kim, H. Y. Rhee, C.-W. Ryu, T. Liu, Y. Wang, and G.-H. Jahng, "Texture analyses of quantitative susceptibility maps to differentiate alzheimer's disease from cognitive normal and mild cognitive impairment," Medical physics, vol. 43, no. 8, pp. 4718-4728, 2016.

[20] Y. Moon, S.-H. Han, and W.-J. Moon, "Patterns of brain iron accumulation in vascular dementia and alzheimers dementia using quantitative susceptibility mapping imaging," Journal of Alzheimer's Disease, vol. 51, no. 3, pp. 737-745, 2016.

[21] M. Azuma, T. Hirai, K. Yamada, S. Yamashita, Y. Ando, M. Tateishi, Y. Iryo, T. Yoneda, M. Kitajima, Y. Wang et al., "Lateral asymmetry and spatial difference of iron deposition in the substantia nigra of patients with parkinson disease measured with quantitative susceptibility mapping," American Journal of Neuroradiology, vol. 37, no. 5, pp. 782-788, 2016.

[22] J. H. O. Barbosa, A. C. Santos, V. Tumas, M. Liu, W. Zheng, E. M. Haacke, and C. E. G. Salmon, "Quantifying brain iron deposition in patients with parkinson's disease using quantitative susceptibility mapping, r2 and r2," Magnetic resonance imaging, vol. 33, no. 5, pp. $559-565,2015$.

[23] G. Du, T. Liu, M. M. Lewis, L. Kong, Y. Wang, J. Connor, R. B. Mailman, and X. Huang, "Quantitative susceptibility mapping of the midbrain in parkinson's disease," Movement Disorders, vol. 31, no. 3 , pp. 317-324, 2016.

[24] X. Guan, M. Xuan, Q. Gu, P. Huang, C. Liu, N. Wang, X. Xu, W. Luo, and M. Zhang, "Regionally progressive accumulation of iron in parkinson's disease as measured by quantitative susceptibility mapping," NMR in Biomedicine, vol. 30, no. 4, 2017.

[25] X. Guan, M. Xuan, Q. Gu, X. Xu, P. Huang, N. Wang, Z. Shen, J. Xu, W. Luo, and M. Zhang, "Influence of regional iron on the motor impairments of parkinson's disease: A quantitative susceptibility mapping study," Journal of Magnetic Resonance Imaging, vol. 45, no. 5, pp. 1335-1342, 2017

[26] N. He, H. Ling, B. Ding, J. Huang, Y. Zhang, Z. Zhang, C. Liu, $\mathrm{K}$. Chen, and F. Yan, "Region-specific disturbed iron distribution in early idiopathic parkinson's disease measured by quantitative susceptibility mapping," Human brain mapping, vol. 36, no. 11, pp. 4407-4420, 2015 .

[27] S. Ide, S. Kakeda, I. Ueda, K. Watanabe, Y. Murakami, J. Moriya, A. Ogasawara, K. Futatsuya, T. Sato, N. Ohnari, K. Okada, A. Matsuyama, H. Fujiwara, M. Hisaoka, S. Tsuji, T. Liu, Y. Wang, and Y. Korogi, "Internal structures of the globus pallidus in patients with parkinson's disease: evaluation with quantitative susceptibility mapping (qsm)," European Radiology, vol. 25, no. 3, pp. 710-718, Mar 2015.

[28] C. Langkammer, L. Pirpamer, S. Seiler, A. Deistung, F. Schweser, S. Franthal, N. Homayoon, P. Katschnig-Winter, M. Koegl-Wallner, T. Pendl, E. M. Stoegerer, K. Wenzel, F. Fazekas, S. Ropele, J. R. Reichenbach, R. Schmidt, and P. Schwingenschuh, "Quantitative susceptibility mapping in parkinson's disease," PLOS ONE, vol. 11, no. 9, pp. 1-13, 092016.

[29] A. K. Lotfipour, S. Wharton, S. T. Schwarz, V. Gontu, A. Schäfer, A. M Peters, R. W. Bowtell, D. P. Auer, P. A. Gowland, and N. P. Bajaj, "High resolution magnetic susceptibility mapping of the substantia nigra in parkinson's disease," Journal of Magnetic Resonance Imaging, vol. 35 , no. 1, pp. 48-55, 2012.

[30] Y. Murakami, S. Kakeda, K. Watanabe, I. Ueda, A. Ogasawara, J. Moriya, S. Ide, K. Futatsuya, T. Sato, K. Okada, T. Uozumi, S. Tsuji, T. Liu, Y. Wang, and Y. Korogi, "Usefulness of quantitative susceptibility mapping for the diagnosis of parkinson disease," American Journal of Neuroradiology, vol. 36, no. 6, pp. 1102-1108, 2015.

[31] A. S. Chandran, M. Bynevelt, and C. R. Lind, "Magnetic resonance imaging of the subthalamic nucleus for deep brain stimulation," Journal of neurosurgery, vol. 124, no. 1, pp. 96-105, 2016.

[32] T. Liu, S. Eskreis-Winkler, A. D. Schweitzer, W. Chen, M. G. Kaplitt, A. J. Tsiouris, and Y. Wang, "Improved subthalamic nucleus depiction with quantitative susceptibility mapping," Radiology, vol. 269, no. 1, pp. 216-223, 2013.

[33] A. Schäfer, B. U. Forstmann, J. Neumann, S. Wharton, A. Mietke, R. Bowtell, and R. Turner, "Direct visualization of the subthalamic nucleus and its iron distribution using high-resolution susceptibility mapping," Human brain mapping, vol. 33, no. 12, pp. 2831-2842, 2012.

[34] J. M. Van Bergen, J. Hua, P. G. Unschuld, I. A. L. Lim, C. K. Jones, R. L. Margolis, C. A. Ross, P. C. Van Zijl, and X. Li, "Quantitative susceptibility mapping suggests altered brain iron in premanifest huntington disease," American Journal of Neuroradiology, vol. 37, no. 5, pp. 789-796, 2016. 
[35] E. Haacke, J. Tang, J. Neelavalli, and Y. Cheng, "Susceptibility mapping as a means to visualize veins and quantify oxygen saturation," Journal of Magnetic Resonance Imaging, vol. 32, no. 3, pp. 663-676, 2010.

[36] K. Kudo, T. Liu, T. Murakami, J. Goodwin, I. Uwano, F. Yamashita, S. Higuchi, Y. Wang, K. Ogasawara, A. Ogawa, and M. Sasaki, "Oxygen extraction fraction measurement using quantitative susceptibility mapping: comparison with positron emission tomography," Journal of Cerebral Blood Flow \& Metabolism, vol. 36, no. 8, pp. 1424-1433, 2016

[37] B. Xu, T. Liu, P. Spincemaille, M. Prince, and Y. Wang, "Flow compensated quantitative susceptibility mapping for venous oxygenation imaging," Magnetic resonance in medicine, vol. 72 , no. 2 , pp. 438 445, 2014.

[38] J. Zhang, T. Liu, A. Gupta, P. Spincemaille, T. D. Nguyen, and Y. Wang, "Quantitative mapping of cerebral metabolic rate of oxygen (cmro2) using quantitative susceptibility mapping (qsm)," Magnetic resonance in medicine, vol. 74, no. 4, pp. 945-952, 2015.

[39] J. Zhang, J. Cho, D. Zhou, T. D. Nguyen, P. Spincemaille, A. Gupta, and Y. Wang, "Quantitative susceptibility mapping-based cerebral metabolic rate of oxygen mapping with minimum local variance," Magnetic Resonance in Medicine, 2017.

[40] J. Zhang, D. Zhou, T. D. Nguyen, P. Spincemaille, A. Gupta, and Y. Wang, "Cerebral metabolic rate of oxygen (cmro2) mapping with hyperventilation challenge using quantitative susceptibility mapping (qsm)," Magnetic resonance in medicine, vol. 77, no. 5, pp. 1762$1773,2017$.

[41] A. P. Fan, B. Bilgic, L. Gagnon, T. Witzel, H. Bhat, B. R. Rosen, and E. Adalsteinsson, "Quantitative oxygenation venography from mri phase," Magnetic resonance in medicine, vol. 72, no. 1, pp. 149-159, 2014.

[42] S. Chang, J. Zhang, T. Liu, A. J. Tsiouris, J. Shou, T. Nguyen, D. Leifer, Y. Wang, and I. Kovanlikaya, "Quantitative susceptibility mapping of intracerebral hemorrhages at various stages," Journal of Magnetic Resonance Imaging, vol. 44, no. 2, pp. 420-425, 2016.

[43] W. Chen, W. Zhu, I. Kovanlikaya, A. Kovanlikaya, T. Liu, S. Wang, C. Salustri, and Y. Wang, "Intracranial calcifications and hemorrhages: characterization with quantitative susceptibility mapping," Radiology, vol. 270 , no. 2, pp. 496-505, 2014

[44] H. Sun, M. Kate, L. C. Gioia, D. J. Emery, K. Butcher, and A. H. Wilman, "Quantitative susceptibility mapping using a superposed dipole inversion method: application to intracranial hemorrhage," Magnetic resonance in medicine, vol. 76, no. 3, pp. 781-791, 2016.

[45] J. Klohs, A. Deistung, F. Schweser, J. Grandjean, M. Dominietto, C. Waschkies, R. M. Nitsch, I. Knuesel, J. R. Reichenbach, and M. Rudin, "Detection of cerebral microbleeds with quantitative susceptibility mapping in the arcabeta mouse model of cerebral amyloidosis," Journal of Cerebral Blood Flow \& Metabolism, vol. 31, no. 12, pp. 2282-2292, 2011.

[46] T. Liu, K. Surapaneni, M. Lou, L. Cheng, P. Spincemaille, and Y. Wang, "Cerebral microbleeds: burden assessment by using quantitative susceptibility mapping," Radiology, vol. 262, no. 1, pp. 269-278, 2012.

[47] W. Liu, K. Soderlund, J. S. Senseney, D. Joy, P.-H. Yeh, J. Ollinger, E. B. Sham, T. Liu, Y. Wang, T. R. Oakes, and G. Riedy, "Imaging cerebral microhemorrhages in military service members with chronic traumatic brain injury," Radiology, vol. 278, no. 2, pp. 536-545, 2015.

[48] A. V. Dimov, Z. Liu, P. Spincemaille, M. R. Prince, J. Du, and Y. Wang, "Bone quantitative susceptibility mapping using a chemical speciesspecific r2* signal model with ultrashort and conventional echo data," Magnetic Resonance in Medicine, 2017.

[49] T. Liu, P. Spincemaille, L. de Rochefort, R. Wong, M. Prince, and Y. Wang, "Unambiguous identification of superparamagnetic iron oxide particles through quantitative susceptibility mapping of the nonlinear response to magnetic fields," Magnetic resonance imaging, vol. 28 , no. 9 , pp. 1383-1389, 2010.

[50] R. Wong, X. Chen, Y. Wang, X. Hu, and M. M. Jin, "Visualizing and quantifying acute inflammation using icam-1 specific nanoparticles and mri quantitative susceptibility mapping," Annals of biomedical engineering, vol. 40, no. 6, pp. 1328-1338, 2012.

[51] L. De Rochefort, R. Brown, M. R. Prince, and Y. Wang, "Quantitative $\mathrm{mr}$ susceptibility mapping using piece-wise constant regularized inversion of the magnetic field," Magnetic Resonance in Medicine, vol. 60, no. 4, pp. 1003-1009, 2008.

[52] B. Kressler, L. De Rochefort, P. Spincemaille, T. Liu, and Y. Wang, "Estimation of sparse magnetic susceptibility distributions from mri using non-linear regularization," ISMRM 2008.

[53] B. Kressler, L. De Rochefort, T. Liu, P. Spincemaille, Q. Jiang, and Y. Wang, "Nonlinear regularization for per voxel estimation of magnetic susceptibility distributions from mri field maps," IEEE transactions on medical imaging, vol. 29, no. 2, p. 273, 2010.

[54] Y. Wang, L. de Rochefort, T. Liu, and B. Kressler, "Magnetic source mri: a new quantitative imaging of magnetic biomarkers," in Engineering in Medicine and Biology Society, 2009. EMBC 2009. Annual International Conference of the IEEE. IEEE, 2009, pp. 53-56.

[55] L. Li and J. S. Leigh, "Quantifying arbitrary magnetic susceptibility distributions with mr," Magnetic resonance in medicine, vol. 51, no. 5, pp. 1077-1082, 2004.

[56] J. Morgan and P. Irarrazaval, "Efficient solving for arbitrary susceptibility distributions using residual difference fields," in Proceedings of International Society for Magnetic Resonance in Medicine, vol. 15, 2007.

[57] E. M. Haacke, N. Y. Cheng, M. J. House, Q. Liu, J. Neelavalli, R. J. Ogg, A. Khan, M. Ayaz, W. Kirsch, and A. Obenaus, "Imaging iron stores in the brain using magnetic resonance imaging," Magnetic resonance imaging, vol. 23 , no. 1 , pp. 1-25, 2005.

[58] K. Shmueli, J. A. de Zwart, P. van Gelderen, T.-Q. Li, S. J. Dodd, and J. H. Duyn, "Magnetic susceptibility mapping of brain tissue in vivo using mri phase data," Magnetic resonance in medicine, vol. 62, no. 6 , pp. 1510-1522, 2009.

[59] H. H. Bauschke and P. L. Combettes, Convex analysis and monotone operator theory in Hilbert spaces. Springer, 2011, vol. 408.

[60] J. Li, S. Chang, T. Liu, Q. Wang, D. Cui, X. Chen, M. Jin, B. Wang, M. Pei, C. Wisnieff, P. Spincemaille, M. Zhang, and Y. Wang, "Reducing the object orientation dependence of susceptibility effects in gradient echo mri through quantitative susceptibility mapping," Magnetic resonance in medicine, vol. 68, no. 5, pp. 1563-1569, 2012.

[61] C. Liu, "Susceptibility tensor imaging," Magnetic resonance in medicine, vol. 63, no. 6, pp. 1471-1477, 2010

[62] F. Schweser, A. Deistung, B. W. Lehr, and J. R. Reichenbach, "Differentiation between diamagnetic and paramagnetic cerebral lesions based on magnetic susceptibility mapping," Medical physics, vol. 37 , no. 10 , pp. $5165-5178,2010$

[63] S. Wharton and R. Bowtell, "Whole-brain susceptibility mapping at high field: a comparison of multiple-and single-orientation methods," Neuroimage, vol. 53, no. 2, pp. 515-525, 2010.

[64] S. Wharton, A. Schäfer, and R. Bowtell, "Susceptibility mapping in the human brain using threshold-based k-space division," Magnetic resonance in medicine, vol. 63, no. 5, pp. 1292-1304, 2010.

[65] W. Li, B. Wu, and C. Liu, "Quantitative susceptibility mapping of human brain reflects spatial variation in tissue composition," Neuroimage, vol. 55, no. 4, pp. 1645-1656, 2011.

[66] C. Liu, W. Li, G. A. Johnson, and B. Wu, "High-field (9.4 t) mri of brain dysmyelination by quantitative mapping of magnetic susceptibility," Neuroimage, vol. 56, no. 3, pp. 930-938, 2011.

[67] T. Liu, I. Khalidov, L. de Rochefort, P. Spincemaille, J. Liu, A. J. Tsiouris, and Y. Wang, "A novel background field removal method for mri using projection onto dipole fields," NMR in Biomedicine, vol. 24, no. 9, pp. 1129-1136, 2011

[68] T. Liu, J. Liu, L. De Rochefort, P. Spincemaille, I. Khalidov, J. R. Ledoux, and Y. Wang, "Morphology enabled dipole inversion (medi) from a single-angle acquisition: comparison with cosmos in human brain imaging," Magnetic resonance in medicine, vol. 66, no. 3, pp. 777-783, 2011.

[69] B. Bilgic, A. Pfefferbaum, T. Rohlfing, E. V. Sullivan, and E. Adalsteinsson, "Mri estimates of brain iron concentration in normal aging using quantitative susceptibility mapping," Neuroimage, vol. 59, no. 3 , pp. 2625-2635, 2012.

[70] C. Langkammer, F. Schweser, N. Krebs, A. Deistung, W. Goessler, E. Scheurer, K. Sommer, G. Reishofer, K. Yen, F. Fazekas, S. Ropele, and J. R. Reichenbach, "Quantitative susceptibility mapping (qsm) as a means to measure brain iron? a post mortem validation study," Neuroimage, vol. 62, no. 3, pp. 1593-1599, 2012.

[71] X. Li, D. S. Vikram, I. A. L. Lim, C. K. Jones, J. A. Farrell, and P. C. van Zijl, "Mapping magnetic susceptibility anisotropies of white matter in vivo in the human brain at $7 \mathrm{t}$," Neuroimage, vol. 62 , no. 1 , pp. 314-330, 2012.

[72] J. Liu, T. Liu, L. de Rochefort, J. Ledoux, I. Khalidov, W. Chen, A. J. Tsiouris, C. Wisnieff, P. Spincemaille, M. R. Prince, and Y. Wang, "Morphology enabled dipole inversion for quantitative susceptibility mapping using structural consistency between the magnitude image and the susceptibility map," Neuroimage, vol. 59, no. 3, pp. 2560 $2568,2012$.

[73] T. Liu, W. Xu, P. Spincemaille, A. S. Avestimehr, and Y. Wang, "Accuracy of the morphology enabled dipole inversion (medi) algorithm 
for quantitative susceptibility mapping in mri," IEEE transactions on medical imaging, vol. 31, no. 3, pp. 816-824, 2012.

[74] F. Schweser, K. Sommer, A. Deistung, and J. R. Reichenbach, "Quantitative susceptibility mapping for investigating subtle susceptibility variations in the human brain," Neuroimage, vol. 62, no. 3, pp. 2083 2100, 2012.

[75] B. Wu, W. Li, A. Guidon, and C. Liu, "Whole brain susceptibility mapping using compressed sensing," Magnetic resonance in medicine, vol. 67, no. 1, pp. 137-147, 2012

[76] F. Schweser, A. Deistung, and J. R. Reichenbach, "Foundations of mri phase imaging and processing for quantitative susceptibility mapping (qsm)," Zeitschrift fuer Medizinische Physik, vol. 26, no. 1, pp. 6-34, 2016.

[77] S. D. Robinson, K. Bredies, D. Khabipova, B. Dymerska, J. P. Marques, and F. Schweser, "An illustrated comparison of processing methods for $\mathrm{mr}$ phase imaging and qsm: combining array coil signals and phase unwrapping," NMR in Biomedicine, vol. 30, no. 4, 2017.

[78] F. Schweser, S. D. Robinson, L. Rochefort, W. Li, and K. Bredies, "An illustrated comparison of processing methods for phase mri and qsm: removal of background field contributions from sources outside the region of interest," NMR in Biomedicine, vol. 30, no. 4, 2017.

[79] J. D. Jackson, Electrodynamics. Wiley Online Library, 1975.

[80] H. A. Lorentz, The Theory of Electrons and Its Applications to the Phenomena of Light and Radiant Heat: A Course of Lectures Delivered in Columbia University, New York, in March and April, 1906. Columbia University Press, 1909, vol. 29.

[81] C. Durrant, M. Hertzberg, and P. Kuchel, "Magnetic susceptibility: further insights into macroscopic and microscopic fields and the sphere of lorentz," Concepts in Magnetic Resonance Part A, vol. 18, no. 1, pp. 72-95, 2003.

[82] L. Evans, Partial Differential Equations, ser. Graduate studies in mathematics. American Mathematical Society, 2010.

[83] E. M. Stein and R. Shakarchi, Functional Analysis: Introduction to Further Topics in Analysis. Princeton University Press, 2011, vol. 4.

[84] J. K. Choi, "Inverse problem in quantitative susceptibility mapping," Ph.D. dissertation, Yonsei University, 2015.

[85] J. K. Choi, H. S. Park, S. Wang, Y. Wang, and J. K. Seo, "Inverse problem in quantitative susceptibility mapping," SIAM Journal on Imaging Sciences, vol. 7, no. 3, pp. 1669-1689, 2014.

[86] J. Tang, S. Liu, J. Neelavalli, Y. Cheng, S. Buch, and E. Haacke, "Improving susceptibility mapping using a threshold-based k-space/image domain iterative reconstruction approach," Magnetic resonance in medicine, vol. 69, no. 5, pp. 1396-1407, 2013.

[87] F. Natterer, "Image reconstruction in quantitative susceptibility mapping," SIAM Journal on Imaging Sciences, vol. 9, no. 3, pp. 1127-1131, 2016

[88] L. Zhou, J. K. Choi, Y. Kee, Y. Wang, and J. K. Seo, "Dipole incompatibility related artifacts in quantitative susceptibility mapping," arXiv preprint arXiv:1701.05457, 2017.

[89] L. Hörmander, The analysis of Linear Partial Differential Operators I, distribution theory and Fourier Analysis, Grundlehren der mathematischen Wissenchaften 256. Springer Verlag, Berlin, 1990.

[90] P. C. Hansen, "Analysis of discrete ill-posed problems by means of the l-curve," SIAM review, vol. 34, no. 4, pp. 561-580, 1992.

[91] —-, Discrete inverse problems: insight and algorithms. SIAM, 2010.

[92] T. F. Chan and J. J. Shen, Image processing and analysis: variational, PDE, wavelet, and stochastic methods. Siam, 2005.

[93] S. Wang, W. Chen, C. Wang, T. Liu, Y. Wang, C. Pan, K. Mu, C. Zhu, $\mathrm{X}$. Zhang, and J. Cheng, "Structure prior effects in bayesian approaches of quantitative susceptibility mapping," BioMed research international, vol. 2016, 2016

[94] S. Wang, T. Liu, W. Chen, P. Spincemaille, C. Wisnieff, A. J. Tsiouris, W. Zhu, C. Pan, L. Zhao, and Y. Wang, "Noise effects in various quantitative susceptibility mapping methods," IEEE Transactions on Biomedical Engineering, vol. 60, no. 12, pp. 3441-3448, 2013.

[95] T. Liu, C. Wisnieff, M. Lou, W. Chen, P. Spincemaille, and Y. Wang, "Nonlinear formulation of the magnetic field to source relationship for robust quantitative susceptibility mapping," Magnetic resonance in medicine, vol. 69, no. 2, pp. 467-476, 2013.

[96] C. Langkammer, K. Bredies, B. A. Poser, M. Barth, G. Reishofer, A. P. Fan, B. Bilgic, F. Fazekas, C. Mainero, and S. Ropele, "Fast quantitative susceptibility mapping using $3 \mathrm{~d}$ epi and total generalized variation," NeuroImage, vol. 111, pp. 622-630, 2015.

[97] K. Bredies, K. Kunisch, and T. Pock, "Total generalized variation," SIAM Journal on Imaging Sciences, vol. 3, no. 3, pp. 492-526, 2010

[98] W. Li, N. Wang, F. Yu, H. Han, W. Cao, R. Romero, B. Tantiwongkosi, T. Q. Duong, and C. Liu, "A method for estimating and removing streaking artifacts in quantitative susceptibility mapping," Neuroimage, vol. 108, pp. 111-122, 2015.

[99] C. B. Poynton, M. Jenkinson, E. Adalsteinsson, E. V. Sullivan, A. Pfefferbaum, and W. Wells III, "Quantitative susceptibility mapping by inversion of a perturbation field model: correlation with brain iron in normal aging," IEEE transactions on medical imaging, vol. 34, no. 1 , pp. 339-353, 2015.

[100] S. Cetin, B. Bilgic, A. Fan, S. Holdsworth, and G. Unal, "Vessel orientation constrained quantitative susceptibility mapping (qsm) reconstruction," in International Conference on Medical Image Computing and Computer-Assisted Intervention. Springer, 2016, pp. 467-474.

[101] L. Bao, X. Li, C. Cai, Z. Chen, and P. C. van Zijl, "Quantitative susceptibility mapping using structural feature based collaborative reconstruction (sfcr) in the human brain," IEEE transactions on medical imaging, vol. 35, no. 9, pp. 2040-2050, 2016.

[102] Y. Kee, J. Cho, K. Deh, Z. Liu, P. Spincemaille, and Y. Wang, "Coherence enhancement in quantitative susceptibility mapping by means of anisotropic weighting in morphology enabled dipole inversion," Magnetic Resonance in Medicine, 2017.

[103] T. Chan, S. Esedoglu, F. Park, and A. Yip, "Recent developments in total variation image restoration," Mathematical Models of Computer Vision, vol. 17, no. 2, 2005

[104] J. Dong, T. Liu, F. Chen, D. Zhou, A. Dimov, A. Raj, Q. Cheng, P. Spincemaille, and Y. Wang, "Simultaneous phase unwrapping and removal of chemical shift (spurs) using graph cuts: application in quantitative susceptibility mapping," IEEE transactions on medical imaging, vol. 34, no. 2, pp. 531-540, 2015.

[105] Z. Liu, Y. Yao, and Y. Wang, "Qsm0—qsm with automatic uniform csf zero reference," ISMRM 2017.

[106] Z. Liu, P. Spincemaille, Y. Yao, Y. Zhang, and Y. Wang, "Qsm0: Quantitative susceptibility mapping with automatic uniform cerebrospinal fluid zero reference," Accepted with revision in Magnetic Resonance in Medicine.

[107] Y. Kee, K. Deh, A. Dimov, P. Spincemaille, and Y. Wang, "Primaldual and forward gradient implementation for quantitative susceptibility mapping," Magnetic Resonance in Medicine, 2017.

[108] M. Maerz, D. Zhou, P. Spincemaille, L. Ruthotto, and Y. Wang, "Image quality improvement using short range finite difference in qsm reconstruction," ISMRM 2015.

[109] G. Aubert and P. Kornprobst, Mathematical problems in image processing: partial differential equations and the calculus of variations. Springer Science \& Business Media, 2006, vol. 147.

[110] C. R. Vogel and M. E. Oman, "Iterative methods for total variation denoising," SIAM Journal on Scientific Computing, vol. 17, no. 1, pp. 227-238, 1996.

[111] T. F. Chan and P. Mulet, "On the convergence of the lagged diffusivity fixed point method in total variation image restoration," SIAM journal on numerical analysis, vol. 36, no. 2, pp. 354-367, 1999.

[112] Y. Saad, Iterative methods for sparse linear systems. SIAM, 2003.

[113] C. R. Vogel, Computational methods for inverse problems. SIAM, 2002.

[114] T. Goldstein and S. Osher, "The split bregman method for 11regularized problems," SIAM Journal on Imaging Sciences, vol. 2, no. 2, pp. 323-343, 2009.

[115] S. Boyd, N. Parikh, E. Chu, B. Peleato, and J. Eckstein, "Distributed optimization and statistical learning via the alternating direction method of multipliers," Foundations and Trends $₫$ in Machine Learning, vol. 3 , no. 1, pp. 1-122, 2011.

[116] W. Yin, S. Osher, D. Goldfarb, and J. Darbon, "Bregman iterative algorithms for \ell_1-minimization with applications to compressed sensing," SIAM Journal on Imaging sciences, vol. 1, no. 1, pp. 143168, 2008.

[117] B. Bilgic, A. P. Fan, J. R. Polimeni, S. F. Cauley, M. Bianciardi, E. Adalsteinsson, L. L. Wald, and K. Setsompop, "Fast quantitative susceptibility mapping with 11-regularization and automatic parameter selection," Magnetic Resonance in Medicine, vol. 72, no. 5, pp. 1444 1459, 2014.

[118] J. Chung and L. Ruthotto, "Computational methods for image reconstruction," NMR in Biomedicine, 2016.

[119] P. Blanchard and E. Brüning, Mathematical Methods in Physics: Distributions, Hilbert Space Operators, Variational Methods, and Applications in Quantum Physics. Birkhäuser, 2015, vol. 69.

[120] L. Ambrosio, N. Fusco, and D. Pallara, Functions of bounded variation and free discontinuity problems. Clarendon Press Oxford, 2000, vol. 254.

[121] A. Chambolle and T. Pock, "A first-order primal-dual algorithm for convex problems with applications to imaging," Journal of Mathemat- 
ical Imaging and Vision, vol. 40, no. 1, pp. 120-145, 2011.

[122] S. Becker, J. Bobin, and E. J. Candès, "Nesta: A fast and accurate firstorder method for sparse recovery," SIAM Journal on Imaging Sciences, vol. 4, no. 1, pp. 1-39, 2011.

[123] M. Jaggi, "Sparse convex optimization methods for machine learning," Ph.D. dissertation, ETH Zürich, 2011

[124] Y. N. Dauphin, R. Pascanu, C. Gulcehre, K. Cho, S. Ganguli, and Y. Bengio, "Identifying and attacking the saddle point problem in high-dimensional non-convex optimization," in Advances in neural information processing systems, 2014, pp. 2933-2941.

[125] G. H. Golub and C. F. Van Loan, Matrix computations. JHU Press, 2012, vol. 3 .

[126] L. N. Trefethen and D. Bau III, Numerical linear algebra. SIAM, 1997, vol. 50.

[127] D. Calvetti, F. Pitolli, E. Somersalo, and B. Vantaggi, "Bayes meets krylov: preconditioning cgls for underdetermined systems," arXiv preprint arXiv:1503.06844, 2015.

[128] S. R. Arridge, M. M. Betcke, and L. Harhanen, "A priorconditioned lsqr algorithm for linear ill-posed problems with edge-preserving regularization," arXiv preprint arXiv:1308.6634, 2013.

[129] D. Zhou, T. Liu, P. Spincemaille, and Y. Wang, "Background field removal by solving the laplacian boundary value problem," NMR in Biomedicine, vol. 27, no. 3, pp. 312-319, 2014.

[130] Z. Liu, Y. Kee, D. Zhou, Y. Wang, and P. Spincemaille, "Preconditioned total field inversion (tfi) method for quantitative susceptibility mapping," Magnetic resonance in medicine, vol. 78, no. 1, pp. 303$315,2017$.

[131] E. M. Haacke, Y. Xu, Y.-C. N. Cheng, and J. R. Reichenbach, "Susceptibility weighted imaging (swi)," Magnetic resonance in medicine, vol. 52, no. 3, pp. 612-618, 2004

[132] M. C. Langham, J. F. Magland, T. F. Floyd, and F. W. Wehrli, "Retrospective correction for induced magnetic field inhomogeneity in measurements of large-vessel hemoglobin oxygen saturation by $\mathrm{mr}$ susceptometry," Magnetic resonance in medicine, vol. 61, no. 3, pp. 626-633, 2009.

[133] Y. Wang, Y. Yu, D. Li, K. Bae, J. Brown, W. Lin, and E. Haacke, "Artery and vein separation using susceptibility-dependent phase in contrast-enhanced mra," Journal of Magnetic Resonance Imaging, vol. 12, no. 5, pp. 661-670, 2000

[134] Y. Wen, D. Zhou, T. Liu, P. Spincemaille, and Y. Wang, "An iterative spherical mean value method for background field removal in mri," Magnetic resonance in medicine, vol. 72, no. 4, pp. 1065-1071, 2014.

[135] H. Sun and A. H. Wilman, "Background field removal using spherical mean value filtering and tikhonov regularization," Magnetic resonance in medicine, vol. 71, no. 3, pp. 1151-1157, 2014.

[136] T. Liu, D. Zhou, P. Spincemaille, and Y. Wang, "Differential approach to quantitative susceptibility mapping without background field removal," ISMRM 2014.

[137] S. Sharma, D. Hernando, D. Horng, and S. Reeder, "A joint background field removal and dipole deconvolution approach for quantitative susceptibility mapping in the liver," ISMRM 2014

[138] B. Bilgic, C. Langkammer, L. L. Wald, and K. Setsompop, "Single-step qsm with fast reconstruction," ISMRM 2014.

[139] I. Chatnuntawech, P. McDaniel, S. F. Cauley, B. A. Gagoski, C. Langkammer, A. Martin, P. E. Grant, L. L. Wald, K. Setsompop, E. Adalsteinsson, and B. Bilgic, "Single-step quantitative susceptibility mapping with variational penalties," NMR in Biomedicine, vol. 30, no. $4,2017$.

[140] L. I. Rudin, S. Osher, and E. Fatemi, "Nonlinear total variation based noise removal algorithms," Physica D: Nonlinear Phenomena, vol. 60, no. 1 , pp. 259-268, 1992 .

[141] T. F. Chan and C.-K. Wong, "Total variation blind deconvolution," IEEE transactions on Image Processing, vol. 7, no. 3, pp. 370-375, 1998.

[142] D. Mumford and J. Shah, "Optimal approximations by piecewise smooth functions and associated variational problems," Communications on pure and applied mathematics, vol. 42, no. 5, pp. 577-685, 1989.

[143] B. K. Horn and B. G. Schunck, "Determining optical flow," Artificial intelligence, vol. 17, no. 1-3, pp. 185-203, 1981.

[144] A. V. Dimov, T. Liu, P. Spincemaille, J. S. Ecanow, H. Tan, R. R. Edelman, and Y. Wang, "Joint estimation of chemical shift and quantitative susceptibility mapping (chemical qsm)," Magnetic resonance in medicine, vol. 73, no. 6, pp. 2100-2110, 2015

[145] S. D. Sharma, D. Hernando, D. E. Horng, and S. B. Reeder, "Quantitative susceptibility mapping in the abdomen as an imaging biomarker of hepatic iron overload," Magnetic resonance in medicine, vol. 74, no. 3, pp. 673-683, 2015

[146] C. Wisnieff, T. Liu, Y. Wang, and P. Spincemaille, "The influence of molecular order and microstructure on the $\mathrm{r} 2^{*}$ and the magnetic susceptibility tensor," Magnetic resonance imaging, vol. 34, no. 5, pp. 682-689, 2016.

[147] W. Li, B. Wu, A. V. Avram, and C. Liu, "Magnetic susceptibility anisotropy of human brain in vivo and its molecular underpinnings," Neuroimage, vol. 59, no. 3, pp. 2088-2097, 2012.

[148] C. Wisnieff, T. Liu, P. Spincemaille, S. Wang, D. Zhou, and Y. Wang, "Magnetic susceptibility anisotropy: cylindrical symmetry from macroscopically ordered anisotropic molecules and accuracy of mri measurements using few orientations," Neuroimage, vol. 70, pp. 363-376, 2013

[149] J. Lounila, M. Ala-Korpela, J. Jokisaari, M. Savolainen, and Y. Kesäniemi, "Effects of orientational order and particle size on the nmr line positions of lipoproteins," Physical review letters, vol. 72, no. 25 , p. 4049,1994

[150] S. Wharton and R. Bowtell, "Fiber orientation-dependent white matter contrast in gradient echo mri," Proceedings of the National Academy of Sciences, vol. 109, no. 45, pp. 18 559-18 564, 2012.

[151] _ "Gradient echo based fiber orientation mapping using $\mathrm{r} 2 *$ and frequency difference measurements," Neuroimage, vol. 83, pp. 1011$1023,2013$.

[152] _ "Effects of white matter microstructure on phase and susceptibility maps," Magnetic resonance in medicine, vol. 73, no. 3, pp. $1258-1269,2015$.

[153] M. Aggarwal, Y. Kageyama, X. Li, and P. C. Van Zijl, "B0-orientation dependent magnetic susceptibility-induced white matter contrast in the human brainstem at $11.7 \mathrm{t}$," Magnetic resonance in medicine, vol. 75 , no. 6, pp. 2455-2463, 2016

[154] X. He and D. A. Yablonskiy, "Biophysical mechanisms of phase contrast in gradient echo mri," Proceedings of the National Academy of Sciences, vol. 106, no. 32, pp. 13 558-13 563, 2009.

[155] J. Luo, X. He, and D. Yablonskiy, "Magnetic susceptibility induced white matter mr signal frequency shiftsexperimental comparison between lorentzian sphere and generalized lorentzian approaches," Mag netic resonance in medicine, vol. 71, no. 3, pp. 1251-1263, 2014

[156] J. Lee, K. Shmueli, M. Fukunaga, P. van Gelderen, H. Merkle, A. C. Silva, and J. H. Duyn, "Sensitivity of mri resonance frequency to the orientation of brain tissue microstructure," Proceedings of the National Academy of Sciences, vol. 107, no. 11, pp. 5130-5135, 2010.

[157] A. L. Sukstanskii and D. A. Yablonskiy, "On the role of neuronal magnetic susceptibility and structure symmetry on gradient echo $\mathrm{mr}$ signal formation," Magnetic resonance in medicine, vol. 71, no. 1, pp. 345-353, 2014.

[158] D. A. Yablonskiy and A. L. Sukstanskii, "Generalized lorentzian tensor approach (glta) as a biophysical background for quantitative susceptibility mapping," Magnetic resonance in medicine, vol. 73, no. 2, pp. 757-764, 2015 